\title{
Alternative dietary protein and water temperature influence the skin and gut microbial communities of yellowtail kingfish (Seriola lalandi)
}

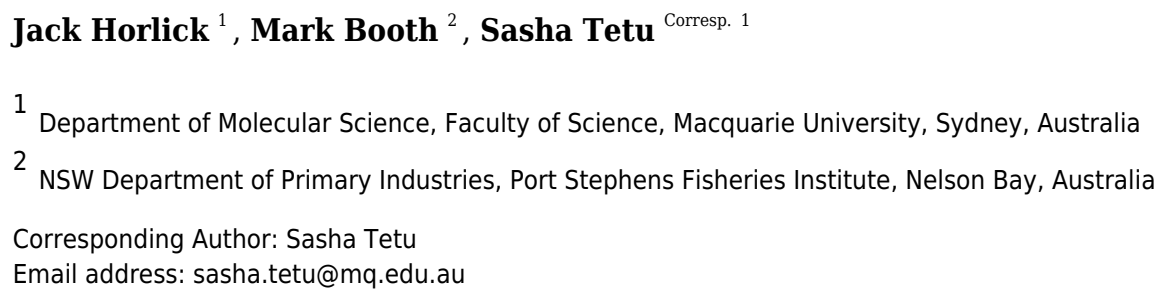

Fish skin and gut microbiomes contribute to host health and growth and are often significantly different in aquaculture-reared fish compared to wild fish. Determining how factors associated with aquaculture, including altered diet and abiotic conditions, affect the microbiome will assist with optimizing farming practices and non-invasively assessing fish health. Here, juvenile yellowtail kingfish (Seriola lalandi) housed at optimal $\left(22^{\circ} \mathrm{C}\right)$ and non-optimal $\left(26^{\circ} \mathrm{C}\right)$ water temperature were fed a fishmeal control diet (FM) or the same diet substituted with $30 \%$ soy-protein concentrate (SPC) in order to investigate impacts on host health and the microbial community composition of the skin mucosa, gut mucosa and digesta. Each of these sites was observed to have a distinct microbiome composition. The combination of SPC and housing at $26^{\circ} \mathrm{C}$ significantly reduced weight gain in yellowtail kingfish and affected immune parameters. The overall microbial composition and relative abundance of specific operational taxonomic units (OTUs) was also significantly altered by inclusion of SPC at $26^{\circ} \mathrm{C}$, with a notable increase in an OTU identified as Photobacterium in the skin mucosa and digesta. Increased relative abundance of Photobacterium sp. was significantly correlated with reduced levels of digesta myeloperoxidase in yellowtail kingfish; a recognized innate immunity defense mechanism. The changes in the microbial communities of yellowtail kingfish fed a diet containing $30 \%$ SPC at $26^{\circ} \mathrm{C}$ highlights the importance of considering the interactive effects of diet and environmental factors on microbiome health in farmed yellowtail kingfish. 
2 Alternative dietary protein and water temperature

3 influence the skin and gut microbial communities of

4 yellowtail kingfish (Seriola lalandi)

Jack Horlick ${ }^{1}$, Mark Booth ${ }^{2}$, Sasha Tetu $^{1}$

7

8

${ }^{1}$ Department of Molecular Science, Faculty of Science, Macquarie University, Sydney, Australia

2 NSW Department of Primary Industries, Port Stephens Fisheries Institute, Nelson Bay, NSW, Australia

Corresponding Author:

Sasha Tetu ${ }^{1}$

NSW Department of Primary Industries, Port Stephens Fisheries Institute, Nelson Bay, NSW, Australia

Email address: sasha.tetu@mq.edu.au

\section{Abstract}

Fish skin and gut microbiomes contribute to host health and growth and are often significantly different in aquaculture-reared fish compared to wild fish. Determining how factors associated with aquaculture, including altered diet and abiotic conditions, affect the microbiome will assist with optimizing farming practices and non-invasively assessing fish health. Here, juvenile yellowtail kingfish (Seriola lalandi) housed at optimal $\left(22^{\circ} \mathrm{C}\right)$ and non-optimal $\left(26^{\circ} \mathrm{C}\right)$ water temperature were fed a fishmeal control diet (FM) or the same diet substituted with $30 \%$ soyprotein concentrate (SPC) in order to investigate impacts on host health and the microbial community composition of the skin mucosa, gut mucosa and digesta. Each of these sites was observed to have a distinct microbiome composition. The combination of SPC and housing at $26^{\circ} \mathrm{C}$ significantly reduced weight gain in yellowtail kingfish and affected immune parameters. The overall microbial composition and relative abundance of specific operational taxonomic units (OTUs) was also significantly altered by inclusion of SPC at $26^{\circ} \mathrm{C}$, with a notable increase in an OTU identified as Photobacterium in the skin mucosa and digesta. Increased relative abundance of Photobacterium sp. was significantly correlated with reduced levels of digesta myeloperoxidase in yellowtail kingfish; a recognized innate immunity defense mechanism. The changes in the microbial communities of yellowtail kingfish fed a diet containing $30 \% \mathrm{SPC}$ at $26^{\circ} \mathrm{C}$ highlights the importance of considering the interactive effects of diet and environmental factors on microbiome health in farmed yellowtail kingfish.

\section{Introduction}


39 Per capita fish consumption has doubled in the last 50 years and aquaculture now provides over

40

41

42

43

44

45

46

47

48

49

50

51

52

53

54

55

56

57

58

59

60

61

62

63

64

65

66

67

68

69

70

71

72

73

74

75

76

77

78

79

80

81 half of all fish for human consumption $(77 \mathrm{~m}$ tonnes in 2015$)(1,2)$. There has been a corresponding increase in the production of aquafeeds and the demand for fishmeal; the traditional source of protein in aquafeeds $(3,4)$. Fishmeal has historically been a key dietary component of the carnivorous farmed fish diet and is generally required in high proportions for optimal health and growth of certain species $(5,6)$. The growth in farming of popular, pelagic carnivores, such as yellowtail kingfish (Seriola lalandi), has substantially contributed to global fishmeal consumption (7). The increasing demand for fishmeal cannot be sustained by wild fishery stocks and therefore diversification of feed sources is an economic and environmental imperative for the aquaculture industry $(7,8)$.

Many plant-based products, including those derived from soybean, have emerged as potential substitutes for fishmeal in a variety of carnivorous and omnivorous fish species (9-11). Methods of raw diet material refinement, such as cooking, grinding or concentration can greatly improve the quality of raw diet materials for fish (e.g. soy protein concentrate), leading to better nutritional outcomes and improved fish health $(12,13)$. However, despite refinement, the dietary inclusion of plant-based feed materials has been linked to gut epithelial damage, reduced nutrient digestibility, and changes in fish immune status which can lead to increased instances of disease such as enteritis $(12,14,15)$. While the cause of these negative outcomes for fish remain unclear, there is evidence that dietary-induced shifts in the fish microbiome are involved (16-19). Indeed, studies in a range of fish species have shown that microbial composition of the gut is significantly influenced by the inclusion of plant-based materials in aquaculture feeds (11, 20-22).

Numerous studies have indicated that the microbiome plays an important role in fish health (see detailed reviews $(19,23-25)$ ). However, our understanding of how diet-induced shifts in the farmed fish microbiome influence digestion, immunity, and the development of gastrointestinal disorders remains incomplete $(6,19)$. Whilst progress has been made in certain species, such as Atlantic salmon (Salmo salar) and rainbow trout (Oncorhynchus mykiss), the effect of diet on the microbiome can vary widely between different fish species $(6,11,17,26)$ and little is known for species in the Seriola genus.

Yellowtail kingfish are an important commercial species both in Australia and worldwide and minimal information is available regarding the microbiome of this species. In larvae, a recent study has shown that changes in diet result in significant shifts in microbial composition and predicted microbial functionality (27). In more mature juveniles, reports are limited to two studies of the digesta microbiome $(28,29)$ and a single study of the gill and skin microbiome $(30)$.

These studies provide a first overview of the skin and digesta microbiomes of yellowtail kingfish, indicating they are dominated by Proteobacteria, as well as varying proportions of Bacteroidetes, Actinobacteria, and Firmicutes, similar to what has been found in other marine fish species $(23,28,30)$. These studies also showed that the digesta microbiome differs between yellowtail kingfish of wild and aquaculture origin and that the skin microbiome is impacted by health status, indicating that the rearing environment, such as water temperature, and diet selection are factors likely to influence the microbial composition of different regions of the fish $(28,30)$. A recent study has also indicated the potential role of specific bacteria in the

Peer] reviewing PDF | (2019:09:41649:1:2:NEW 13 Jan 2020) 
82 yellowtail kingfish microbiome in preventing microbial disease (31).To our knowledge, there are

83

84

85

86

87

88

89

90

91

92

93

94

95

96

97

98

99

100

101

102

103

104

105

106

107

108

109

110

111

112

113

114

115

116

117

118

119

120

121

122

123

124

no published studies to date investigating the gut mucosal microbiome of yellowtail kingfish.

In this work we examined the effects of inclusion of fishmeal (FM) and soy protein concentrate (SPC) on the microbiome of juvenile yellowtail kingfish reared at optimal $\left(22^{\circ} \mathrm{C}\right)$ and non-optimal $\left(26^{\circ} \mathrm{C}\right)$ water temperature. We analyzed how shifts in the microbial composition of skin, gut and digesta of yellowtail kingfish were correlated with measured changes in selected biometric responses and immune-related health parameters. We also provide a detailed overview of the microbial composition of juvenile yellowtail kingfish across multiple body sites, substantially increasing what is known about the microbiome of this commercially important fish species.

\section{Materials \& Methods}

\section{Overview of experiment}

Triplicate groups of yellowtail kingfish were fed a control diet based on fish meal (FM) or the same diet substituted with $30 \%$ soy protein concentrate (SPC). A $30 \%$ substitution was chosen as it has been previously shown that soy inclusion above this ratio significantly impacts growth in juvenile yellowtail kingfish, with the driver of reduced growth remaining unknown (14). Each diet was fed to fish at two water temperatures; an optimal temperature of $22^{\circ} \mathrm{C}$ and a supraoptimal temperature of $26^{\circ} \mathrm{C}$ The optimal and non-optimal rearing temperature were based upon previous work by Pirozzi and Booth (32). The orthogonal combination of diet type and water temperature resulted in four treatments; hereafter $22 \mathrm{FM}$ and three experimental treatments 22 SPC, 26 FM, and 26 SPC). The experiment was done at the NSW Department of Primary Industries (NSW DPI) Port Stephens Fisheries Institute (Taylors Beach, 2316 NSW, Australia) in accordance with laws and ethics requirements regarding nutrition research with fish as overseen by the Department of Primary Industries (Fisheries) Animal Care and Ethics Committee (NSW DPI ACEC Authority 93/5).

\section{Composition of experimental diets}

The FM control diet was composed mainly of prime fishmeal (68\%) and wheat flour $(27 \%)$ with a small amount of fish oil (3.5\%). The SPC diet was made by blending the FM mash and SPC in a 70:30 ratio; however, supplements such choline chloride, vitamin- $C$ and vitamin-mineral premix were kept constant between diets. The crude protein and gross energy content of the both FM and SPC diets were approximately $58 \%$ and $20.8 \mathrm{MJ} \mathrm{kg}^{-1}$, respectively. The formulation, proximate composition and amino acid composition of the diets are detailed in Supplementary Table 1. All raw diet materials were supplied by Ridley Aquafeed Pty Ltd (Robart Court, Narangba, 4504 QLD, Australia) and 8mm diameter pellets were manufactured using a twinscrew extruder (MPF24:25, Baker Perkins, Peterborough, United Kingdom), oven dried at $45^{\circ} \mathrm{C}$ until moisture content was $<12 \%$, cooled and stored frozen $\left(-17^{\circ} \mathrm{C}\right)$. Diets were manufactured at the CSIRO Bribie Island Research Centre (Woorim, 4507 QLD, Australia).

\section{Feeding protocols}


125 Mixed sex yellowtail kingfish were obtained from the NSW DPI Marine Fish Hatchery at PSFI

126

127

128

129

130

131

132

133

134

135

136

137

138

139

140

141

142

143

144

145

146

147

148

149

150

151

152

153

154

155

156

157

158

159

160

161

162

163

164

165

166

167

168 and on-grown at low densities in a recirculating aquaculture system. Immediately prior to the experiment fish were being fed a $6 \mathrm{~mm}$ commercial aquafeed (Skretting Australia, Cambridge, 7170 TAS, Australia). A schematic representation of the experimental flow-through systems used to conduct the feeding trial is detailed in Supplementary Figure 1. Briefly, each flowthrough system was supplied with raw estuarine water obtained from the Tilligerry Creek adjacent PSFI. This water was firstly filtered through a series of sand filters, a cartridge particle filter and finally two $5 \mu \mathrm{m}$ bag filters before being transferred to separate supply tanks. Each flow-through system consisted of two 10,000 L supply tanks (storing filtered water) fitted with refrigerated temperature control units and an oxygen diffuser. Temperature controlled water from each supply tank was pumped at constant rate of 6-7L $\mathrm{min}^{-1}$ to six $200 \mathrm{~L}$ independent cylindrical polythene tanks. All effluent water was directed to waste.

Seven fish, graded from a larger population, were allocated to each $200 \mathrm{~L}$ tank ensuring consistent tank biomass (individual fish weight $=245 \pm 18 \mathrm{~g}$ and tank biomass $=1,719 \mathrm{~g} \pm 32 \mathrm{~g}$ ). Fish were individually fin-clipped on stocking for subsequent identification. Fish were then gradually acclimated to the temperature regimes over eight days and were handfed a $6 \mathrm{~mm}$ commercial aquafeed (Skretting, Australia) once daily (11:00 hours) at 2\% body weight. This ration was slightly restrictive to ensure that all feed was consumed, resulting in equal feed across all treatments. On day nine, fish were lightly anesthetized (10 mg L-1, AQUI-S尺), identified by fin-clip, and individually measured for fork length and weight. Two fish from each tank were selected at this time for baseline health and microbiome data. Each diet was then randomly assigned to triplicate experimental tanks in each temperature system. Fish were handfed test diets once daily (11:00 hours) at 3\% of the measured tank biomass for the first for 9 days. After 10 days a new feed amount was calculated for each tank based on the expected weight gain of fish and a feed conversion ratio (FCR) of 1.0. This change was based on growth data published on juvenile yellowtail kingfish reared on soy-based diets (14). The same procedure was adopted to increase the quantum of feed required for the remaining 11 days of the trial. The trial was concluded after 21 days at which time all remaining fish were sampled. Rearing water quality parameters were recorded twice daily and are detailed in Supplementary Table 2.

\section{Sampling procedures}

Microbial communities were sampled from the skin mucosa (skin), distal digesta (digesta), and adhered distal gut mucosa (gut mucosa) of individual animals. Each fish was transferred to a sterilized stainless-steel benchtop and euthanized by lkejime. The skin mucus from the right side of the fish between the pectoral and caudal fin was gently scraped with a sterile scalpel blade and the mucus transferred to a sterile $1.5 \mathrm{~mL}$ tube and immediately stored on ice. Blood was drawn from the caudal vein at the base of the tail using a $23 \mathrm{G}$ sterile hypodermic needle and transferred to a $1 \mathrm{~mL}$ K3E KEDTA Minicollect tube (Grenier Bio-one) and immediately stored on ice. The fork length and weight of the animal was then recorded before the exterior surfaces were sterilized $100 \%$ ethanol. The fish was dissected and the intestine and viscera were carefully removed. The fish, less viscera, and the liver were individually weighed. The distal gut digesta was aseptically excised into a sterile $1.5 \mathrm{~mL}$ tube through gently squeezing the 
169

170

171

172

173

174

175

176

177

178

179

180

181

182

183

184

185

186

187

188

189

190

191

192

193

194

195

196

197

198

199

200

201

202

203

204

205

206

207

208

209

210

211

212

gut so as not to include the mucosa and immediately stored on ice. The intestine was opened lengthways and gently washed in sterile phosphate buffered saline $(0.01 \mathrm{M})$ to remove nonadhered luminal gut contents. The gut mucosa was gently scraped using a sterile scalpel blade and the mucosa transferred to a sterile $1.5 \mathrm{~mL}$ tube before immediate storage on ice. The microbiota samples were then transferred to and stored in a $-80^{\circ} \mathrm{C}$ freezer until analyzed. Blood samples were centrifuged at 11,300 rpm for $14 \mathrm{~min}$ and the plasma transferred to a sterile 1.5 $\mathrm{mL}$ tube and stored at $-20^{\circ} \mathrm{C}$ until further analysis. A $45 \mathrm{~mL}$ aliquot of filtered input estuarine water, in replicate, was sampled on the final day of the trial and stored at $-80^{\circ} \mathrm{C}$ until further analysis.

\section{Plasma lysozyme activity}

Plasma lysozyme activity was determined by a turbidimetric assay utilizing lyophilized Micrococcus lysodeikticus cells (Sigma, Australia) using a method modified from (33). Lysozyme from chicken egg white (Sigma, Australia) was used as a standard. In a 96 well plate, $15 \mu \mathrm{L}$ of plasma samples diluted 1:40 in $0.02 \mathrm{M}$ sodium citrate buffer were added to $150 \mu \mathrm{L}$ of M. lysodeikticus suspended in $0.02 \mathrm{M}$ sodium citrate buffer at a concentration of $0.2 \mathrm{mg} / \mathrm{mL}$. The absorbance was immediately measured at $450 \mathrm{~nm}$, and subsequent measurements were taken every $5 \mathrm{~min}$ for $60 \mathrm{~min}$. A unit of lysozyme activity was defined as the quantity of plasma required to reduce absorbance by $0.001 / \mathrm{min}[111]$.

\section{Digesta myeloperoxidase activity}

The myeloperoxidase (MPO) activity of digesta samples was determined using a colorimetric assay utilizing 3,3',5,5'- tetramethylbenzidine hydrochloride (TMB) (Sigma, Australia), following the method of Quade and Roth (1995) with minor modifications (34). To prepare equal solutions of digesta, $3 \mu \mathrm{l}$ of $0.02 \mathrm{M}$ sodium citrate buffer was added for each $\mathrm{mg}$ of sample (c. $250 \mathrm{mg}$ ). Samples were heated to $55^{\circ} \mathrm{C}$ for two min and briefly vortexed and this was repeated twice Samples were centrifuged at $3000 \mathrm{xg}$ for $5 \mathrm{~min}$ and the supernatant collected for further analysis. Five $\mu \mathrm{L}$ of digesta supernatant were added to $95 \mu \mathrm{L}$ of Hanks' Balanced Salt Solution (HBSS) without Ca2+ and Mg2+ (Thermo Fisher Scientific, Australia) in a 96 well plate. Next, $35 \mu \mathrm{L}$ of freshly prepared $20 \mathrm{mM} \mathrm{TMB}$ and $5 \mathrm{mM} \mathrm{H}_{2} \mathrm{O}_{2}$ was added to each well and the reaction stopped after 2 min by addition of $35 \mu \mathrm{L}$ of $4 \mathrm{M}$ sulphuric acid. The optical density was read at $450 \mathrm{~nm}$.

\section{DNA extraction and sequencing}

DNA extractions were performed using the FastDNA spin kit (MP Biomedicals, Australia) with additional isopropanol purification steps, following the method of Hart and colleagues (2015) (35). Water samples were filtered through a Sterivex ${ }^{\mathrm{TM}}$ GP $0.22 \mu \mathrm{m}$ filter unit (Millipore, Australia) to isolate the bacteria from the water. The filter was removed and processed in the same manner as the other microbiome samples.

Following extraction, the V4 region of the 16S rRNA gene was amplified using 515 forward and 806 reverse primers with custom barcodes, based upon the earth microbiome primer protocols $(36,37)$. PCR amplification was performed on 1:50 dilutions of extracted DNA using MyFi Mix (Bioline, Australia) with a primer concentration of $400 \mathrm{nM}$ in a final volume of $30 \mu \mathrm{L}$. Samples were PCR amplified with 35 cycles at $95^{\circ} \mathrm{C}$ for 15 seconds, $50^{\circ} \mathrm{C}$ for 15 seconds and $72^{\circ} \mathrm{C}$ for 
21360 seconds. Previously isolated bacterial genomic DNA was used as a positive control and 214 DNAse free water as a negative control. Samples were quantified using Quant-iT ${ }^{\mathrm{TM}}$ PicoGreen $\circledast$ 215 (Invitrogen, Australia). Barcoded amplicons were pooled at equimolar concentrations and gel 216 purified using the Wizard® SV gel and PCR clean up system (Promega, Australia). Pooled 217 amplicons were sequenced using the Illumina MiSeq platform (MiSeq V2 $2 \times 250$ bp paired-end 218 sequencing run) at the Ramaciotti Centre for Genomics, Sydney, Australia.

219

220

221

222

223

224

225

226

227

228

229

230

231

232

233

234

235

236

237

238

239

240

241

242

243

244

245

246

247

248

249

250

251

252

253

254

255

PCR assay to screen for plpV \& sequencing of PCR products

A region of the plpV gene was amplified using primers described by Vences and colleagues (2017) (38). PCR amplification was performed on 1:50 dilutions of extracted DNA using MyFi Mix (Bioline, Australia) with a primer concentration of $400 \mathrm{nM}$ in a final volume of $30 \mu \mathrm{L}$. Samples were PCR amplified with 35 cycles at $95^{\circ} \mathrm{C}$ for 30 seconds, $54.5^{\circ} \mathrm{C}$ for 30 seconds and $72^{\circ} \mathrm{C}$ for 60 seconds. Five microliters of resulting amplicons were visualized on $2 \%$ agarose gels (Bioline, Australia) using a $1 \mathrm{kbp}$ Hyperladder (Bioline, Australia). Three samples were selected to confirm the identity of the amplified product, with Sanger sequencing of bands gel purified using a Wizard® SV gel and PCR clean up system (Promega, Australia), carried out by Macrogen (South Korea). Sequence identity was checked by blastn and blastx searches of the NCBI nucleotide and protein databases using Geneious 11.1.5 (https://www.geneious.com) (39, 40).

\section{Bioinformatics and statistical analyses}

Raw sequences were demultiplexed by the Ramaciotti Centre for Genomics, Sydney, Australia. Demultiplexed sequences were processed using Quantitative Insights Into Microbial Ecology 2 (QIIME2) software (version 2018.4) (41). Quality control was performed within QIIME2 using DADA2 (42). No truncation of the forward or reverse reads was required based upon the quality scores. Taxonomy was assigned using the QIIME2 q2-feature-classifier plugin and a Naïve Bayes classifier that was trained on the SILVA 99\% OTU database trimmed to the V4 region of the $16 \mathrm{~S}$ rRNA gene $(41,43)$. Samples with a total number of reads less than 10,000 were discarded from further analysis. OTUs from mitochondria and chloroplasts were removed. Rarefaction plots of samples have been included in Supplementary Figure 2.

Statistical calculations and graphical construction analysis of amplicon sequence data were performed in the RStudio statistical package (version 1.1.453). Alpha diversity analyses were performed in the phyloseq R package (V.1.24.0) using multifactor analysis of variance (ANOVA) followed by a post hoc Tukey honest significant difference test (44). Non-metric multidimensional scaling (nMDS) plots were constructed using the phyloseq $R$ package (V.1.24.0) (44). The phyloseq R package (V.2.5-2) was used to perform permutational multivariate analysis of variance (PERMANOVA) with 999 permutations on Bray Curtis distance matrices (V.1.24.0) and to normalize the data used to produce Venn diagrams (45). Differential abundance of OTUs between treatments were identified using the linear discriminant analysis (LDA) effect size (LEfSe) method, available at http://huttenhower.sph.harvard.edu/galaxy/ (46). OTU relative abundance with treatments as the classes of subjects was used as the input. Alpha values of 0.05 were used for the factorial

Peer) reviewing PDF | (2019:09:41649:1:2:NEW 13 Jan 2020) 
256

257

258

259

260

261

262

263

264

265

266

267

268

269

270

271

272

273

274

275

276

277

278

279

280

281

282

283

284

285

286

287

288

289

290

291

292

293

294

295

296

297

298

Kruskal-Wallis sum test and the pairwise Wilcoxen test between classes. A threshold of 2.0 was chosen for logarithmic LDA scores.

Spearman correlations, corrected for multiple inference using Holm's method, and node weightings for network analysis were calculated using R package Hmisc (rcorr.adjust) (47). Correlations were considered significant when the correlation $p$-value was $<0.05$. Significant correlations were visualised in Cytoscape v.3.6.1 (48).

Significant differences between treatments for the biometric responses were determined by twoway ANOVA. The fixed factors were diet type (FM vs SPC) and water temperature $\left(22^{\circ} \mathrm{C}\right.$ vs $\left.26^{\circ} \mathrm{C}\right)$. Each treatment was applied in triplicate. If ANOVA proved significant $(p<0.05)$ a Tukeys honestly significant difference test was used to separate the treatment means. Statistical analysis was done using GraphPad Prism (version 7) software (GraphPad Software, USA).

\section{Data deposition}

The 16S rRNA gene sequence data generated and analyzed in this study can be found in the GenBank Sequence Read Archive (SRA) database under accession number SUB4557405.

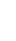

\section{Results}

\section{Analyses of biometric responses and immune parameters}

Triplicate groups of Yellowtail kingfish were fed fishmeal (FM) and soy protein concentrate $(\mathrm{SPC})$ based diets at optimal $\left(22^{\circ} \mathrm{C}\right)$ and non-optimal $\left(26^{\circ} \mathrm{C}\right)$ temperatures. The orthogonal combination of diet type and water temperature resulted in four treatments; hereafter a control treatment (22 FM) and three experimental treatments (22 SPC, 26 FM and 26 SPC). The survival rate of fish across all treatments was $100 \%$ and no obvious signs of illness were noted on internal and external examination of sampled specimens. Specific growth rate (weight) of fish, shown in Table 1, was found to be significantly affected by both water temperature ( $p=$ $0.0017, F=21.24)$ and by diet $(p=0.049, F=5.36)$; however, there was no interaction between the water temperature and diet factors $(p=0.87, F=0.03)$ when analyzed by two way ANOVA. Relative weight gain was significantly lower in fish reared at $26^{\circ} \mathrm{C}$, irrespective of the diet fish were fed (Table 1). An increase (worsening) in feed conversion ratio (FCR) of was observed for all experimental treatments compared to $22 \mathrm{FM}$, but was only significant for 26 SPC (Table 1). Feed conversion ratio was calculated as feed given (g) divided by weight gain (g). No adjustment was made for waste as all feed was consumed. Fulton's condition factor and the hepatosomatic index of fish were not significantly affected by water temperature and diet or their interaction (Table 1).

Assays were performed to measure components of the innate immune system in digesta and plasma samples. Digesta myeloperoxidase (MPO), which is produced by neutrophils, was significantly lower (one way ANOVA; $p=0.12 \& \mathrm{~F}=1.055$ ) in fish fed $\mathrm{SPC}$ at $22^{\circ} \mathrm{C}$ and $26^{\circ} \mathrm{C}$ compared to fish fed the $22 \mathrm{FM}$ control treatment (Figure 1-A). Plasma lysozyme levels were significantly higher in fish fed SPC at $26^{\circ} \mathrm{C}$ compared to fish fed the FM diet at $22^{\circ} \mathrm{C}$ (Figure 1- 
299 B). However, the 26 SPC treatment was not significantly different to the groups fed SPC at $22^{\circ} \mathrm{C}$

300

301

302

303

304

305

306

307

308

309

310

311

312

313

314

315

316

317

318

319

320

321

322

323

324

325

326

327

328

329

330

331

332

333

334

335

336

337

338

339

340

341 or $\mathrm{FM}$ at $26^{\circ} \mathrm{C}$ (Figure 1-B).

\section{Microbiome sequencing and assignment of OTUs}

A total of 84 juvenile yellowtail kingfish were sampled resulting in 48 gut mucosal samples, 59 digesta samples and 55 skin mucosal samples following quality filtering (Table 2). DNA extractions were performed on an additional 36 gut mucosal samples, 25 digesta samples and 29 skin mucosal samples; however, these did not yield visible PCR products or failed to yield reads upon sequencing. Fish body site samples had on average $\geq 47,556$ reads, while water samples had an average of 33,371 reads following sequence quality control and filtering (Table 2 ). A total of 7,787 unique sequence variants were detected, which were assigned to 1,713 OTUs' at a $99 \%$ similarity level.

Pairwise permutation multivariate analysis of variance tests (PERMANOVA) confirmed that there were no significant differences between the baseline microbial community profiles of different tanks of fish at the beginning of the experiment (Supplementary Table 3).

PERMANOVA showed no significant influence of tank on the microbial composition within each treatment for any of the body sites, indicating that tank was not a significant factor driving community changes (Supplementary Table 4).

\section{Comparison of microbial composition across body sites}

Microbiome alpha diversity measures were calculated for each of the body sites and the water. The microbial community richness (Chao1) and diversity (Simpson and Shannon) observed in the skin samples were significantly higher than that of the gut mucosa and digesta. There was no significant difference in the alpha diversity measures between the digesta and gut mucosa (Figure 2). The alpha diversity measures in the rearing water were more similar to the skin than the internal gut microbiome samples (Figure 2).

Of the 1,713 OTUs identified, the rearing water shared 288 OTUs with the skin, while there were 274 OTUs in common between water and gut mucosa and 239 shared with the digesta and only 5 OTUs which were specific to the rearing water (Figure 3). The skin contained the highest number of unique OTUs (985) with digesta and gut mucosa sites containing only 112 and 84 unique OTUs respectively. The skin and gut mucosa housed 750 common OTUs, while the digesta microbiome shared somewhat lower numbers of OTUs with the other sites (590 OTUS shared with skin and 558 OTUs in common with gut mucosa microbiomes). Of the core microbiome observed across all body sites (260 OTUs), 53\% were assigned as Proteobacteria, $15 \%$ as Firmicutes, $14 \%$ as Actinobacteria, $8 \%$ as Bacteroidetes, $2 \%$ as Cyanobacteria, with the remaining $8 \%$ split between 16 additional phyla.

Microbial community composition was observed to be strongly influenced by body site (PERMANOVA analysis, Table 3). The community composition of the skin was significantly different from that of the gut mucosa and digesta in all four treatments (Table 3). Based on PERMANOVA, the gut mucosa and digesta bacterial communities were not significantly 
342 different in fish fed FM diets. However, in fish fed SPC diets, the gut mucosa and digesta

343

344

345

346

347

348

349

350

351

352

353

354

355

356

357

358

359

360

361

362

363

364

365

366

367

368

369

370

371

372

373

374

375

376

377

378

379

380

381

382

383

384

385 microbial communities were significantly different (Table 3).

\section{Skin microbiome response to altered diet and water temperature}

The overall community microbial composition of the skin samples was affected by both diet and water temperature. We observed significant differences in the skin microbiomes of fish fed FM and SPC diets, based on PERMANOVA analysis (Table 4). Elevated water temperature also affected the microbial communities, with a significant difference between the $22 \mathrm{FM}$ control treatment and the 26 FM treatments (Table 4). The combination of elevated water temperature and inclusion of SPC had the greatest impact on the skin microbiome, as this treatment was associated with the strongest shift in community composition compared to the $22 \mathrm{FM}$ control treatment (Table 4).

The skin microbiome in all treatments was dominated by four phyla, which together contributed a combined relative abundance of $>90 \%$. These phyla, in order of relative abundance, were Proteobacteria, Bacteroidetes, Firmicutes, and Actinobacteria (Supplementary Figure 3). At the genus level only four genera contributed $>5 \%$ of relative abundance of the skin microbiome: Ralstonia, Photobacterium, unassigned bacterium of the family Rhodobacteraceae, and Thalassotalea (Figure 4). Approximately $35 \%$ of the inferred microbial population in each treatment was assigned to these four genera. The remaining population was comprised of 949 lower abundance genera, of which 916 had a relative abundance of less than $1 \%$.

To investigate which OTUs significantly contributed to the differences in skin microbiome composition associated with diet and temperature, differential abundance testing with linear discriminant analysis (LDA), effect size (LEfse) analysis was performed. We found that 25, 23, and 12 OTUs were significantly differentially abundant in the 22 SPC, 26 FM, and 26 SPC treatments respectively when compared to the control treatment (Figure 5). For all three treatments, the relative abundance of OTUs assigned to Photobacterium and Lawsonella were increased when compared to the control treatment, while OTUs assigned to Chitinophagales and Salegentibacter were signficantly decreased. The 22 SPC treatment was associated with increased abundance of several OTUs that were not significantly increased in the other treatments, including Acinetobacter, Streptococcus, Haemophilus, and Micrococcus representatives (Figure 5). The 26 SPC treatment was associated with increased levels of only two OTUs, assigned as Lawsonella and Photobacterium, whereas the 22 SPC and 26 FM treatments were associated with higher levels of 21 and 6 OTUs respectively when compared to the control treatment. While these OTUs were found to be significantly differentially abundant between treatments, considerable variability was still observed in the response of individuals within the treatments (Figure 5). However, the variability of individuals within each treatment did not appear to be impacted by which tank fish were housed (Figure 5).

\section{Digesta microbiome response to altered diet and water temperature}

As with the skin, the digesta microbial communities of fish fed SPC at $22^{\circ} \mathrm{C}$ were significantly different from the $22 \mathrm{FM}$ control when analysed by PERMANOVA (Table 5). Similar differences were noted in fish housed at $26^{\circ} \mathrm{C}$, which had significantly altered community profiles compared 
386

387

388

389

390

391

392

393

394

395

396

397

398

399

400

401

402

403

404

405

406

407

408

409

410

411

412

413

414

415

416

417

418

419

420

421

422

423

424

425

426

427

428

429 to the $22 \mathrm{FM}$ control treatment (Table 5). As with the skin, the most pronounced shift in digesta community composition from the control treatment was noted in fish fed SPC at $26^{\circ} \mathrm{C}$ (Table 5).

Digesta microbial communities were dominated by the family Vibrionaceae, with a relative abundance of over $80 \%$ across all four treatments (Figure 6 ). No other family comprised more than $5 \%$ of the population in any treatment. Whilst the relative abundance of the family Vibrionaceae was relatively consistent across treatments, specific genera within this family were strongly impacted by both diet and temperature, with an OTU assigned to the genus Photobacterium dominating the communities across the experimental treatments relative to the control treatment (Figure 6). To establish how the digesta microbiome changes in respect to altered diet and elevated temperature, the set of OTUs showing significant differences between treatments was determined. LEfse analysis revealed 5, 3, and 10 differentially abundant OTUs in the 22 SPC, 26 FM, and 26 SPC treatments respectively when compared to the control treatment (Figure 7). Across each of the three experimental treatments, the relative abundance of OTU 1622, assigned to genus Photobacterium, was significantly increased with respect to the control treatment (Figure 7). The combination of $26^{\circ} \mathrm{C}$ and SPC diet was associated with significantly lower abundance of nine OTUs (relative to the control treatment), whereas the 22 SPC and 26 FM treatments were both associated with a decrease in one OTU (Figure 7). Within each treatment there was no common trend in the relative abundance of OTUs for each tank, indicating that the housing tank was not a major driver of the differences in microbial composition between samples (Figure 7).

OTU 1622, assigned to genus Photobacterium, was dominated (94\%) by a single sequence variant. A blastN search of the NCBI nucleotide database (July 2018) showed this sequence variant shares $100 \%$ identity only with characterized strains of species Photobacterium damselae, including Photobacterium damselae subsp. damselae (GenBank ID MG077071.1) and Photobacterium damselae subsp. piscicida (GenBank ID MH472944.1) which have been reported as fish pathogens $(49,50)$. Screening for the $P$. damselae haemolytic phospholipase gene plpV, a marker of virulent strains of this species [1], was carried out using the 43 endpoint digesta samples. All 38 digesta samples that had a relative abundance of OTU 1622 greater than $20 \%$ were PCR positive for this marker, while no PCR product was observed in the 5 samples with a relative abundance of OTU 1622 less than $2 \%$. Sequence confirmation performed on three positive samples showed that all shared $>96 \%$ nucleotide identity and $100 \%$ translated amino acid identity with PlpV in Photobacterium damselae subsp. piscicida and Photobacterium damselae subsp. piscicida (Supplementary Table 5, 6). A blastN search of the NCBI nucleotide database (July 2018) of the representative sequences of OTUs 1619 and 1626 (both also assigned to the family Vibrionaceae) did not provide a reliable indication of taxonomy at a more detailed level as both sequences matched a number of different Vibrio species.

Network analysis was performed to determine how specific OTUs correlated with diet, temperature, and measured health and growth parameters. Higher abundance of OTU 1622, tentatively thought to represent pathogenic Photobacterium damselae, was significantly correlated with lower levels of digesta MPO (Figure 8). OTU 1622 was also significantly positively correlated with the SPC diet (and negatively with FM diet) (Figure 8). OTU 1622 was

Peer] reviewing PDF | (2019:09:41649:1:2:NEW 13 Jan 2020) 
430 the only OTU that was significantly correlated with diet $(p<0.05)$. There was a positive

431

432

433

434

435

436

437

438

439

440

441

442

443

444

445

446

447

448

449

450

451

452

453

454

455

456

457

458

459

460

461

462

463

464

465

466

467

468

469

470

471

472

association between the FM diet and higher levels of digesta MPO. Fourteen OTUs were positively correlated with increased levels of digesta MPO (Figure 8). The most abundant of these was OTU 1619 , classified to family Vibrionaceae, which had a maximum relative abundance of $66 \%$ across all digesta samples. The other 13 OTUs all had a relative abundance of less than $4 \%$ in any given sample and were diverse in their taxonomic classification, spanning seven different taxonomic orders (Supplementary Table 7).

\section{Gut mucosa microbiome response to altered diet and water temperature}

Compared to the skin and digesta communities, the gut mucosa microbiome was found to be less affected by alterations in diet and temperature. The overall gut mucosa microbial community showed no significant differences between the treatments when analyzed by PERMANOVA (Supplementary Table 8). In all treatments, the gut mucosa microbiome was dominated by three genera of the family Vibrionaceae (Figure 9-A). These three genera had a combined relative abundance of $91 \%$ in $22 \mathrm{FM}, 89 \%$ in $22 \mathrm{SPC}, 87 \%$ in $26 \mathrm{FM}$, and $76 \%$ in 26 SPC treatments (Figure 9-A). The only other genus that comprised over $2 \%$ of the population in any of the treatments was Ralstonia, with the remainder of the population assigned to 264 genera with an abundance of less than $2 \%$ (Figure 9-A). While there were clear differences in the mean relative abundance of different genera by treatment, individuals within each treatment showed a high degree of variability in genera level relative abundance (Figure 9-B). LEfse analysis revealed only one OTU that had a significantly different relative abundance between treatments. OTU 1619, which is only classified to the family level of Vibrionaceae was statistically significantly lower in the 22 SPC (LDA -5.5), 26 FM (LDA -5.4), and 26 SPC (LDA 5.2) treatments.

\section{Discussion}

\section{Diet and temperature impact growth and innate immunity}

The dietary inclusion of $30 \%$ SPC had a significant impact on the growth of juvenile yellowtail kingfish. This effect was amplified at an elevated water temperature of $26^{\circ} \mathrm{C}$. Similar impacts on weight gain were noted in another study feeding SPC to juvenile yellowtail kingfish (14). Previous studies have shown that the reduced digestibility of soy-based diets can impact the growth and feed conversion ratio of farmed fish (51). Whilst digestibility was not measured in the current experiment, the feed conversion ratio of the FM and SPC diets at optimal temperature $\left(22^{\circ} \mathrm{C}\right)$ were comparable, indicating that digestibility was likely not a key factor. Irrespective of water temperature, the inclusion of SPC was also associated with changes in immune parameters. Digesta myeloperoxidase (MPO), a component of the fish innate immune system produced by neutrophils (52), was found to be at significantly lower levels in fish fed SPC when compared to fish fed a FM based diet. In contrast, plasma lysozyme levels were increased in fish fed the SPC diet. Plasma lysozyme is an immune defense molecule that causes lysis of bacteria through breaking linkages in the cell wall (53). Increased levels of lysozyme activity have also been observed in Atlantic salmon (Salmo salar) fed diets containing SPC (54).

\section{Microbial composition and diversity of sampled yellowtail kingfish body sites}

Peer] reviewing PDF | (2019:09:41649:1:2:NEW 13 Jan 2020) 
473 The overall microbial community composition of the skin mucosa was distinct from the other 474 sampled sites and exhibited significantly higher alpha diversity. Prior work in rainbow trout 475 (Oncorhynchus mykiss) and Atlantic salmon has also shown the skin microbiome to be distinct 476 from that of the gut (55-57) and higher diversity in the skin compared to gut is consistent with 477 previous work in rainbow trout (55). The skin alpha diversity metrics seen in our study are also 478 consistent with previous findings for yellowtail kingfish (30); however, the alpha diversity of the 479 digesta microbiota was not as low as reported here. It is noteworthy that a recent study 480 investigating the gut microbiome of wild Atlantic cod (Gadus morhua) also found similarly low 481 levels of gut diversity and reported that the microbiome was dominated by an OTU assigned as 482 Photobacterium (58). It is possible, therefore, that the genus Photobacterium is able to rapidly 483 484 colonize and become dominant in the gut of certain fish species.

Proteobacteria were predominant among the set of OTUs common to all sites followed by varying proportions of Bacteroidetes, Firmicutes, and Actinobacteria. This is broadly consistent with previous yellowtail kingfish microbiome research where these four phyla were found to be highly abundant in both the skin and digesta microbiomes $(28,30)$. Work in other farmed fish species has also found Proteobacteria to be the dominant phylum in the microbiome, followed by differing proportions of Firmicutes, Actinobacteria, and Bacteroidetes (57, 59-61). At genus level gut mucosal and digesta communities were dominated by OTUs assigned to Vibrio and Photobacterium. These two genera are reported to be dominant members of carnivorous fish microbiomes across numerous studies, as reviewed recently by Egerton and colleagues (2018), although it is worth noting $16 \mathrm{~S}$ rRNA gene copy is relatively high for these genera, which could contribute to some extent to their high relative abundance. (24). In contrast, Soriano and colleagues (2018) did not note high proportions of these genera in yellowtail kingfish reared in saltwater recirculation systems at $22^{\circ} \mathrm{C}$ or at $26^{\circ} \mathrm{C}(29)$. This discrepancy highlights the substantial difficulty in obtaining consistent microbial populations across experimental systems, even within the same species and applying similar community analysis methodology.

The skin microbiome is affected by changes in diet and water temperature The skin mucosa of yellowtail kingfish was associated with 982 unique OTUs and shared only 92 OTUs with the estuarine water samples, indicating that the skin microbiome is independent of the surrounding water (Figure 4). This finding is consistent with that of Chiarello and colleagues (2015) who noted that the skin microbial communities of both gilthead seabream (Sparus aurata) and European seabass (Dicentrarchus labrax) shared few OTUs with the water (59).

\section{8}

The skin microbiome community composition was significantly altered by both water temperature and diet treatments. Temperature variation is well known to affect bacterial growth rates and particular species or strains may have differences in their optimal growth temperature, however specific temperature preferences are only known for some culturable isolates. The relative abundance of OTUs assigned to Photobacterium and Lawsonella were increased in the 515 Lawsonella belongs to the family Corynebacteriaceae and increases in the abundance of this 516 family have also been previously reported in response to altered diet (62). Rimoldi and 
517 colleagues noted that the relative abundance of Corynebacteriaceae was increased in the gut of 518 rainbow trout fed diets containing high proportions of animal by-product compared to fishmeal 519 diets, indicating that alternative feeds may specifically impact this family (62). Low levels of

520 Photobacterium have been noted in past microbiome surveys of the skin of marine fish; 521 however, this genus showed higher relative abundance in this study than previously reported $522(30,57,59,63)$. The relative abundance of Photobacterium was particularly high in the $26 \mathrm{FM}$, 52322 SPC and 26 SPC treatments, indicating that raw diet material selection and temperature can 524 influence the level of these bacteria on the skin. These alterations may be as a result of diet and temeprature induced alterations in the composition of mucins in the skin mucosa which have previously been shown to influence the microbiome and are a key component of this mucosal barrier $(19,64,65)$. Diet and temperature related changes in the microbiome may also be due to

528

529

530

531

532 altered composition of mucosal immunoglobulins, which potentially play a key role in determining which bacterial species inhabit the mucosa (66-68).

\section{The skin microbiome as a potential non-invasive biomarker of fish health}

533

534 Elevated temperature, regardless of diet, was associated with a decrease in the relative abundance of OTUs assigned to Alteromonadaceae when compared to the control. Lower relative abundance of Alteromonadaceae has previously been associated with poor health, with

535

536

537 lower levels observed in yellowtail kingfish exhibiting enteritis and in Atlantic salmon infected with Salmonid alphavirus $(30,69)$. It should also be noted that Soriano and colleagues (2018), whom made no note of reduced health in yellowtail kingfish fish reared at $26^{\circ} \mathrm{C}$, did not observe altered levels of Alteromonadaceae (29). Given that fish housed at $26^{\circ} \mathrm{C}$ in the present study were associated with lower growth and altered immune parameters, the relative abundance of

540

541 Alteromonadaceae in the skin might be a potential indicator of poor fish performance and

542 reduced health status; however, further work would be needed to determine this. Increased prevalence of the genus Photobacterium in the skin community might also be used as an indicator of health, possibly in conjunction with Alteromonadaceae to non-invasively assess the health status of fish.

545

546

547

\section{Diet and temperature influence the microbiome of the digesta}

548 The inclusion of SPC at $22^{\circ} \mathrm{C}$ resulted in a substantial increase in the relative abundance of

549 OTU 1622 (assigned to Photobacterium) in the digesta relative to the $22 \mathrm{FM}$ control along with increases in three other bacterial OTUs assigned to Clostridiales and Streptococcus. The combination of SPC at $26^{\circ} \mathrm{C}$ was also associated with an increase in OTU1622, while nine OTUs were found to be significantly reduced in their relative abundance compared to the

552

553

554 control.

555

Analysis of the sequence variants within OTU 1622 indicates that this likely represents the species $P$. damselae, but does not distinguish between different strains of this species which are reported to differ in their pathogenicity (38). Detection of the plpV virulence gene in all digesta samples for which OTU 1622 was abundant (>20\% of relative abundance) indicates that this OTU is potentially a representative of pathogenic $P$. dameselae. This suggests that dietary inclusion of SPC, particularly in combination with elevated water temperature may create opportunities for pathogenic strains of $P$. damselae to dominate in the digesta, out-competing 
561

562

563

564

565

566

567

568

569

570

571

572

573

574

575

576

577

578

579

580

581

582

583

584

585

586

587

588

589

590

591

592

593

594

595

596

597

598

599

600

601

602

603

604

other microbial species. It should be noted that there are multiple genes that contribute to pathogenicity in $P$. damselae (38) and therefore the presence of $p / p V$ is not conclusive evidence for pathogenicity, especially given there were no obvious sign of photobaceteriosis in the fish at the end of this trial. Longer-term experiments involving culturing and characterization of predominant digesta bacteria would be useful for further investigating the connection between diet, $P$. damselae abundance and yellowtail kingfish health.

Network analysis showed that high relative abundance of OTU 1622 was correlated with reduced MPO in the digesta, indicating that this OTU may be influencing MPO production in the gut. Virulent strains of $P$. damselae ssp. piscicida have been shown to induce apoptosis of sea bass (Dicentrarchus labrax $L$.) neutrophils, and therefore it is possible that the reduction in measurable MPO is due to Photobacterium induced apoptosis of neutrophils (49). However, we were not able to determine whether the observed alterations in the levels of MPO are mediated by changes in the microbiome or are due to a direct impact of the diets we tested. Soy-based diets have previously been shown to induce alterations in the histology of the gut, and therefore may be directly influencing neutrophil recruitment and thus the level of $\operatorname{MPO}(12,70)$. It is also possible that a combination of increased relative abundance of OTU 1622 and the direct impact of SPC on the gut both acted to influence the levels of MPO in the digesta. The correlation between reduced levels of MPO and significantly higher relative abundance of OTU 1622 indicates that suppression of the fish immune system through these intimated mechanisms, or indeed other mechanisms, may have contributed to the dominance of the genus Photobacterium observed in the digesta. Further studies considering a wider range of immune parameters are required to follow up on these findings.

\section{The gut mucosal microbiome is less responsive to changes in diet and temperature}

Unlike the digesta microbiome, the composition of the gut mucosa microbiome was not strongly affected by the SPC diet or altered water temperature (Supplementary Table 8). For gut mucosal samples the relative abundance of OTU 1622 (assigned to Photobacterium) did not reach the same levels as in the digesta and no significantly differentially abundant OTUs were observed for any of the treatments. The microbiota of the digesta are considered free-living and transient, in contrast to the gut mucosal community, which has undergone long term coevolution with the host and is influenced to a greater degree by host physiology (25). Indeed, the chemical composition of the gut mucosal layer plays a key role in bacterial adhesion and therefore their ability to colonise the mucosa (25). It is possible that the gut mucosa microbiota may be slower to respond to the particular perturbations used in this work and a longer term study may show additional changes in this community.

\section{Conclusions}

Many previous studies investigating fish microbiomes have involved surveying populations of wild fish and farmed fish $(23,28,30)$. Such studies provide an overview of the microbial communities and can provide insight into how different factors contribute to shaping the microbiome, with environment and diet often being implicated as drivers of microbial

Peer) reviewing PDF | (2019:09:41649:1:2:NEW 13 Jan 2020) 
605 composition. However, given there are many differences between the lifestyles of wild and

606 farmed yellowtail kingfish it is has not been possible to determine the degree to which specific

607 factors are responsible for influencing or controlling the composition of the microbiome. Here,

608 we performed a controlled study which measured the separate and combined effects of diet and

609 water temperature on the microbiome of yellowtail kingfish. We observed distinct changes in

610 microbial community profiles as well as significant alterations in fish growth and specific immune

611 parameters. Given that the microbiome in fish has been shown to influence digestion, nutrient

612 assimilation, and stimulation of the immune system in other studies, we suggest that high levels

613 of SPC $(30 \%)$ and elevated water temperature $\left(26^{\circ} \mathrm{C}\right)$ resulted in shifts in the microbial

614 communities of juvenile yellowtail kingfish which subsequently influenced their growth trajectory

615 and immune status $(66,71)$.

616

617 In the digesta of fish fed the SPC diet at $26^{\circ} \mathrm{C}$, a single OTU tentatively identified as pathogenic

$618 P$. damselae, dominated the microbial community. This OTU was correlated with a reduction in

619 levels of the innate immune defense molecule MPO. Given the significantly lower growth

620 observed in this group, as well as the alterations to their digesta microbiome, we recommend

621 limiting the amount of SPC in commercial diets for yellowtail kingfish until further inclusion

622 studies are undertaken.

623

624 An elevated water temperature of $26^{\circ} \mathrm{C}$ independently influenced the response of the yellowtail

625 kingfish microbiome to changes in diet and also further contributed to lower growth rates. These

626 results indicate that the impact of stressors commonly associated with commercial fish

627 production (such as non-optimal water temperature) should be carefully considered by

628 aquaculture practitioners before they commence widespread use of alternative dietary materials

629 or they make significant changes to the diet of their fish. Many factors such as water

630 temperature are not normally controllable in a commercial fish farm, therefore the seasonal

631 timing of dietary changes will likely be important. Future studies of the impact of alternative raw

632 diet materials would benefit from considering both optimal and non-optimal environmental

633 conditions encountered in commercial aquaculture settings. This might include examination of

634 changing abiotic factors such as salinity, $\mathrm{pH}$ and dissolved oxygen.

635

636

637 Acknowledgements \& author contributions

638 We thank the all the technicians at the Port Stephens Fisheries Institute, including Justin

639 Tierney, Ian Russel, Steve Gamble and Basseer Codabaccus, for all their assistance with the

640 feeding trial. We would also like to thank the NSW DPI for access to the facilities and resources

641 of the Port Stephens Fisheries Institute. We thank Ridley Aquafeed (Michael Salaini) for

642 providing raw materials used to make the diets.

643

644 We thank Dr Hasinika Gamage, Dr Liam Elbourne, and Prof lan Paulsen for their assistance

645 and technical support with the bioinformatic analysis.

646 We declare that we have no competing financial or nonfinancial interests.

647 
648 J.H. contributed to the experimental design, feeding trial, laboratory work, bioinformatics, 649 statistics and manuscript writing. M.B. contributed to the experimental design, feeding trial, 650 statistics, and manuscript corrections. S.T. contributed to the experimental design, laboratory 651 work, bioinformatics, statistics, and manuscript corrections.

652

653

654

655

656

657

658

659

660

661

662

663

664

665

666

667

668

669

670

671

672

673

674

675

676

677

678

679

680

681

682

683

684

685

686

687

688

689

690

691

692

693

\section{References}

1. FAO. 2017. FAO Global Aquaculture Summary Information 2015. FAO Fisheries and Aquaculture Department, Rome, Italy.

2. FAO. 2016. The State of World Fisheries and Aquaculture 2016. Rome $200 \mathrm{pp}$.

3. Cao L, Naylor R, Henriksson P, Leadbitter D, Metian M, Troell M, Zhang W. 2015.

China's aquaculture and the world's wild fisheries. Science 347:133-135.

4. Klinger D, Naylor R. 2012. Searching for solutions in aquaculture: charting a sustainable course. Annual Review of Environment and Resources 37:247-276.

5. Bendiksen EÅ, Johnsen CA, Olsen HJ, Jobling M. 2011. Sustainable aquafeeds: Progress towards reduced reliance upon marine ingredients in diets for farmed Atlantic salmon (Salmo salar L.). Aquaculture 314:132-139.

6. Ringø E, Zhou Z, Vecino JLG, Wadsworth S, Romero J, Krogdahl A, Olsen RE, Dimitroglou A, Foey A, Davies S, Owen M, Lauzon HL, Martinsen LL, Schryver PD, Bossier P, Sperstad S, Merrifield DL. 2016. Effect of dietary components on the gut

microbiota of aquatic animals. A never-ending story? Aquaculture Nutrition 22:219-282

7. Kobayashi M, Msangi S, Batka M, Vannuccini S, Dey MM, Anderson JL. 2015. Fish to 2030: The role and opportunity for aquaculture. Aquaculture Economics \& Management 19:282-300.

8. Tacon AGJ, Metian M. 2015. Feed matters: Satisfying the feed demand of aquaculture. Reviews in Fisheries Science \& Aquaculture 23:1-10.

9. Kissinger KR, García-Ortega A, Trushenski JT. 2016. Partial fish meal replacement by soy protein concentrate, squid and algal meals in low fish-oil diets containing

10. Hartviksen M, Bakke AM, Vecino JG, Ringø E, Krogdahl Å. 2014. Evaluation of the effect of commercially available plant and animal protein sources in diets for Atlantic salmon (Salmo salar L.): digestive and metabolic investigations. Fish Physiology and Biochemistry 40:1621-1637.

11. Zhou Z, Ringø E, Olsen RE, Song SK. 2018. Dietary effects of soybean products on gut microbiota and immunity of aquatic animals: A review. Aquaculture Nutrition 24:644-665.

12. Bansemer MS, Forder REA, Howarth GS, Suitor GM, Bowyer J, Stone DAJ. 2015. The effect of dietary soybean meal and soy protein concentrate on the intestinal mucus layer and development of subacute enteritis in Yellowtail Kingfish (Seriola lalandi) at suboptimal water temperature. Aquaculture Nutrition 21:300-310.

13. Stone DAJ, Bellgrove EJ, Forder REA, Howarth GS, Bansemer MS. 2017. Inducing Subacute Enteritis in Yellowtail Kingfish Seriola lalandi: the Effect of Dietary Inclusion of Soybean Meal and Grape Seed Extract on Hindgut Morphology and Inflammation. North American Journal of Aquaculture 80:59-68. 
694

695

696

697

698

699

700

701

702

703

704

705

706

707

708

709

710

711

712

713

714

715

716

717

718

719

720

721

722

723

724

725

726

727

728

729

730

731

732

733

734

735

736

737

738

739

740

741

742

743

14. Bowyer JN, Qin JG, Smullen RP, Adams LR, Thomson MJS, Stone DAJ. 2013. The use of a soy product in juvenile yellowtail kingfish (Seriola lalandi) feeds at different water temperatures: 2. Soy protein concentrate. Aquaculture 410-411:1-10.

15. Hardy RW. 2010. Utilization of plant proteins in fish diets: effects of global demand and supplies of fishmeal. Aquaculture Research 41:770-776.

16. Bruce TJ, Neiger RD, Brown ML. 2018. Gut histology, immunology and the intestinal microbiota of rainbow trout, Oncorhynchus mykiss (Walbaum), fed process variants of soybean meal. Aquaculture Research 49:492-504.

17. Schmidt V, Amaral-Zettler L, Davidson J, Summerfelt S, Good C. 2016. Influence of fishmeal-free diets on microbial communities in Atlantic Salmon (Salmo salar) recirculation aquaculture systems. Applied and Environmental Microbiology 82:44704481.

18. Gajardo K, Jaramillo-Torres A, Kortner TM, Merrifield DL, Tinsley J, Bakke AM, Krogdahl A. 2017. Alternative protein sources in the diet modulate microbiota and functionality in the distal intestine of Atlantic salmon (Salmo salar). Applied and Environmental Microbiology 83:e02615-16.

19. Legrand TPRA, Wynne JW, Weyrich LS, Oxley APA. 2019. A microbial sea of possibilities: current knowledge and prospects for an improved understanding of the fish microbiome. Reviews in Aquaculture n/a.

20. Desai AR, Links MG, Collins SA, Mansfield GS, Drew MD, Van Kessel AG, Hill JE. 2012. Effects of plant-based diets on the distal gut microbiome of rainbow trout (Oncorhynchus mykiss). Aquaculture 350:134-142.

21. Estruch G, Collado M, Peñaranda D, Vidal AT, Cerdá MJ, Martínez GP, MartinezLlorens S. 2015. Impact of fishmeal replacement in diets for gilthead sea bream (Sparus Aurata) on the gastrointestinal microbiota determined by pyrosequencing the 16S rRNA gene. PloS one 10:e0136389.

22. Green TJ, Smullen R, Barnes AC. 2013. Dietary soybean protein concentrate-induced intestinal disorder in marine farmed Atlantic salmon, Salmo salar is associated with alterations in gut microbiota. Veterinary Microbiology 166:286-292.

23. Tarnecki AM, Burgos FA, Ray CL, Arias CR. 2017. Fish intestinal microbiome: diversity and symbiosis unravelled by metagenomics. Journal of Applied Microbiology 123:2-17.

24. Egerton S, Culloty S, Whooley J, Stanton C, Ross RP. 2018. The Gut Microbiota of Marine Fish. Frontiers in Microbiology 9:873-873.

25. Banerjee G, Ray AK. 2017. Bacterial symbiosis in the fish gut and its role in health and metabolism. Symbiosis 72:1-11.

26. Wang AR, Ran C, Ringø E, Zhou ZG. 2017. Progress in fish gastrointestinal microbiota research. Reviews in Aquaculture 10:626-640.

27. Wilkes Walburn J, Wemheuer B, Thomas T, Copeland E, O'Connor W, Booth M, Fielder S, Egan S. 2019. Diet and diet-associated bacteria shape early microbiome development in Yellowtail Kingfish (Seriola lalandi). Microbial Biotechnology 12:275-288.

28. Ramírez C, Romero J. 2017. The microbiome of Seriola lalandi of wild and aquaculture origin reveals differences in composition and potential function. Frontiers in Microbiology 8.

29. Soriano EL, Ramírez DT, Araujo DR, Gómez-Gil B, Castro LI, Sánchez CG. 2018. Effect of temperature and dietary lipid proportion on gut microbiota in yellowtail kingfish Seriola lalandi juveniles. Aquaculture 497:269-277.

30. Legrand TPRA, Catalano SR, Wos-Oxley ML, Stephens F, Landos M, Bansemer MS, Stone DAJ, Qin JG, Oxley APA. 2018. The inner workings of the outer surface: Skin and gill microbiota as indicators of changing gut health in yellowtail kingfish. Frontiers in Microbiology 8.

Peer] reviewing PDF | (2019:09:41649:1:2:NEW 13 Jan 2020) 
744 31. Ramírez C, Rojas R, Romero J. 2019. Partial Evaluation of Autochthonous Probiotic

745

746

747

748

749

750

751

752

753

754

755

756

757

758

759

760

761

762

763

764

765

766

767

768

769

770

771

772

773

774

775

776

777

778

779

780

781

782

783

784

785

786

787

788

789

790

791

792

793

794

32. Pirozzi I, Booth MA. 2009. The routine metabolic rate of mulloway (Argyrosomus japonicus: Sciaenidae) and yellowtail kingfish (Seriola lalandi: Carangidae) acclimated to six different temperatures. Comparative Biochemistry and Physiology Part A: Molecular \& Integrative Physiology 152:586-592.

33. Cha S-H, Lee J-S, Song C-B, Lee K-J, Jeon Y-J. 2008. Effects of chitosan-coated diet on improving water quality and innate immunity in the olive flounder, Paralichthys olivaceus. Aquaculture 278:110-118.

34. Quade MJ, Roth JA. 1997. A rapid, direct assay to measure degranulation of bovine neutrophil primary granules. Veterinary Immunology and Immunopathology 58:239-48.

35. Hart ML, Meyer A, Johnson PJ, Ericsson AC. 2015. Comparative evaluation of DNA extraction methods from feces of multiple host species for downstream next-generation sequencing. PLoS ONE 10:e0143334.

36. Gilbert JA, Jansson JK, Knight R. 2014. The Earth Microbiome project: successes and aspirations. BMC Biology 12:69.

37. Caporaso JG, Lauber CL, Walters WA, Berg-Lyons D, Lozupone CA, Turnbaugh PJ, Fierer N, Knight R. 2011. Global patterns of 16S rRNA diversity at a depth of millions of sequences per sample. Proceedings of the National Academy of Sciences 108:45164522.

38. Vences A, Rivas AJ, Lemos ML, Husmann M, Osorio CR. 2017. Chromosome-encoded hemolysin, phospholipase, and collagenase in plasmidless isolates of Photobacterium damselae subsp. damselae contribute to virulence for fish. Applied and Environmental Microbiology 83:e00401-17.

39. Altschul SF, Gish W, Miller W, Myers EW, Lipman DJ. 1990. Basic local alignment search tool. Journal of Molecular Biology 215:403-10.

40. States DJ, Gish W. 1994. Combined use of sequence similarity and codon bias for coding region identification. Journal of Computational Biology 1:39-50.

41. Caporaso JG, Kuczynski J, Stombaugh J, Bittinger K, Bushman FD, Costello EK, Fierer N, Pena AG, Goodrich JK, Gordon JI, Huttley GA, Kelley ST, Knights D, Koenig JE, Ley RE, Lozupone CA, McDonald D, Muegge BD, Pirrung M, Reeder J, Sevinsky JR, Turnbaugh PJ, Walters WA, Widmann J, Yatsunenko T, Zaneveld J, Knight R. 2010. QIIME allows analysis of high-throughput community sequencing data. Nature Methods 7:335-336.

42. Callahan BJ, McMurdie PJ, Rosen MJ, Han AW, Johnson AJA, Holmes SP. 2016. DADA2: High-resolution sample inference from Illumina amplicon data. Nature Methods 13:581.

43. Quast C, Pruesse E, Yilmaz P, Gerken J, Schweer T, Yarza P, Peplies J, Glöckner FO. 2013. The SILVA ribosomal RNA gene database project: improved data processing and web-based tools. Nucleic Acids Research 41:D590-D596.

44. McMurdie PJ, Holmes S. 2013. phyloseq: An r package for reproducible interactive analysis and graphics of microbiome census data. PLoS ONE 8:e61217.

45. Dixon P. 2009. VEGAN, a package of $R$ functions for community ecology. Journal of Vegetation Science 14:927-930.

46. Afgan E, Baker D, van den Beek M, Blankenberg D, Bouvier D, Čech M, Chilton J, Clements D, Coraor N, Eberhard C, Grüning B, Guerler A, Hillman-Jackson J, Von Kuster G, Rasche E, Soranzo N, Turaga N, Taylor J, Nekrutenko A, Goecks J. 2016. The Galaxy platform for accessible, reproducible and collaborative biomedical analyses: 2016 update. Nucleic Acids Research 44:W3-W10.

47. Harrell F. 2010. Hmisc: Harrell miscellaneous. R package version 3.7-0.

PeerJ reviewing PDF | (2019:09:41649:1:2:NEW 13 Jan 2020) 
795

796

797

798

799

800

801

802

803

804

805

806

807

808

809

810

811

812

813

814

815

816

817

818

819

820

821

822

823

824

825

826

827

828

829

830

831

832

833

834

835

836

837

838

839

840

841

842

843

844

48. Shannon P, Markiel A, Ozier O, Baliga NS, Wang JT, Ramage D, Amin N, Schwikowski B, Ideker T. 2003. Cytoscape: A Software Environment for Integrated Models of Biomolecular Interaction Networks. Genome Research 13:2498-2504.

49. do Vale A, Marques F, Silva MT. 2003. Apoptosis of sea bass (Dicentrarchus labrax L.) neutrophils and macrophages induced by experimental infection with Photobacterium damselae subsp. piscicida. Fish \& Shellfish Immunology 15:129-144.

50. Xie ZY, Hu CQ, Zhang LP, Chen C, Ren CH, Shen Q. 2007. Identification and pathogenicity of Vibrio ponticus affecting cultured Japanese sea bass, Lateolabrax japonicus Letters in Applied Microbiology 45:62-7.

51. Deng J, Mai K, Ai Q, Zhang W, Wang X, Xu W, Liufu Z. 2006. Effects of replacing fish meal with soy protein concentrate on feed intake and growth of juvenile Japanese flounder, Paralichthys olivaceus. Aquaculture 258:503-513.

52. Palić D, Andreasen CB, Menzel BW, Roth JA. 2005. A rapid, direct assay to measure degranulation of primary granules in neutrophils from kidney of fathead minnow (Pimephales promelas Rafinesque, 1820). Fish \& Shellfish Immunology 19:217-227.

53. Saurabh S, Sahoo PK. 2008. Lysozyme: an important defence molecule of fish innate immune system. Aquaculture Research 39:223-239.

54. Krogdahl, Bakke-Mckellep, RØed, Baeverfjord. 2000. Feeding Atlantic salmon Salmo salar $L$. soybean products: effects on disease resistance (furunculosis), and lysozyme and IgM levels in the intestinal mucosa. Aquaculture Nutrition 6:77-84.

55. Lowrey L, Woodhams DC, Tacchi L, Salinas I. 2015. Topographical mapping of the rainbow trout (Oncorhynchus mykiss) microbiome reveals a diverse bacterial community with antifungal properties in the skin. Applied and Environmental Microbiology 81:69156925.

56. Gajardo K, Rodiles A, Kortner TM, Krogdahl Å, Bakke AM, Merrifield DL, Sørum H. 2016. A high-resolution map of the gut microbiota in Atlantic salmon (Salmo salar): A basis for comparative gut microbial research. Scientific Reports 6:30893.

57. Minniti G, Hagen LH, Porcellato D, Jørgensen SM, Pope PB, Vaaje-Kolstad G. 2017. The Skin-Mucus Microbial Community of Farmed Atlantic Salmon (Salmo salar). Frontiers in Microbiology 8.

58. Riiser ES, Haverkamp THA, Borgan Ø, Jakobsen KS, Jentoft S, Star B. 2018. A single vibrionales $16 \mathrm{~S}$ rRNA oligotype dominates the intestinal microbiome in two geographically separated atlantic cod populations. Frontiers in Microbiology 9.

59. Chiarello M, Villéger S, Bouvier C, Bettarel Y, Bouvier T. 2015. High diversity of skinassociated bacterial communities of marine fishes is promoted by their high variability among body parts, individuals and species. FEMS Microbiology Ecology 91:61.

60. Larsen A, Tao Z, Bullard SA, Arias CR. 2013. Diversity of the skin microbiota of fishes: evidence for host species specificity. FEMS Microbiology Ecology 85:483-494.

61. Uren Webster TM, Consuegra S, Hitchings M, Garcia de Leaniz C. 2018. Interpopulation variation in the Atlantic salmon microbiome reflects environmental and genetic diversity. Applied and Environmental Microbiology 84.

62. Rimoldi S, Terova G, Ascione C, Giannico R, Brambilla F. 2018. Next generation sequencing for gut microbiome characterization in rainbow trout (Oncorhynchus mykiss) fed animal by-product meals as an alternative to fishmeal protein sources. PLoS ONE 13:e0193652.

63. Siriyappagouder P, Galindo-Villegas J, Lokesh J, Mulero V, Fernandes JMO, Kiron V. 2018. Exposure to yeast shapes the intestinal bacterial community assembly in zebrafish larvae. Frontiers in Microbiology 9.

64. Merrifield DL, Rodiles A. 2015. The fish microbiome and its interactions with mucosal tissues, p 273-295, Mucosal health in aquaculture. Elsevier.

PeerJ reviewing PDF | (2019:09:41649:1:2:NEW 13 Jan 2020) 
845 65. Tapia-Paniagua ST, Ceballos-Francisco D, Balebona MC, Esteban MÁ, Moriñigo MÁ.

846

847

848

849

850

851

852

853

854

855

856

857

858

859

860

861

862

863

864

865

866 2018. Mucus glycosylation, immunity and bacterial microbiota associated to the skin of experimentally ulcered gilthead seabream (Sparus aurata). Fish \& Shellfish Immunology 75:381-390.

66. Cecelia K, Irene S. 2017. Under pressure: interactions between commensal microbiota and the teleost immune system. Frontiers in Immunology 8.

67. Gomez D, Sunyer JO, Salinas I. 2013. The mucosal immune system of fish: The evolution of tolerating commensals while fighting pathogens. Fish \& Shellfish Immunology 35:1729-1739.

68. Xu Z, Parra D, Gómez D, Salinas I, Zhang Y-A, von Gersdorff Jørgensen L, Heinecke RD, Buchmann K, LaPatra S, Sunyer JO. 2013. Teleost skin, an ancient mucosal surface that elicits gut-like immune responses. Proceedings of the National Academy of Sciences of the United States of America 110:13097-13102.

69. Reid KM, Patel S, Robinson AJ, Bu L, Jarungsriapisit J, Moore LJ, Salinas I. 2017. Salmonid alphavirus infection causes skin dysbiosis in Atlantic salmon (Salmo salar L.) post-smolts. PLoS ONE 12:e0172856.

70. Bakke-McKellep AM, Penn MH, Salas PM, Refstie S, Sperstad S, Landsverk T, Ringø E, Krogdahl A. 2007. Effects of dietary soyabean meal, inulin and oxytetracycline on intestinal microbiota and epithelial cell stress, apoptosis and proliferation in the teleost Atlantic salmon (Salmo salar L.). British Journal of Nutrition 97:699-713.

71. Ray AK, Ghosh K, Ringø E. 2012. Enzyme-producing bacteria isolated from fish gut: a review. Aquaculture Nutrition 18:465-492.

867 
Figure 1

Levels of (A) digesta MPO and (B) plasma lysozyme in yellowtail kingfish for each treatment.

Boxplot with points representing individual samples and the box representing the median and interquartile range. Different letters denote statistically significant $(p<0.05)$ differences between treatments by one way ANOVA.
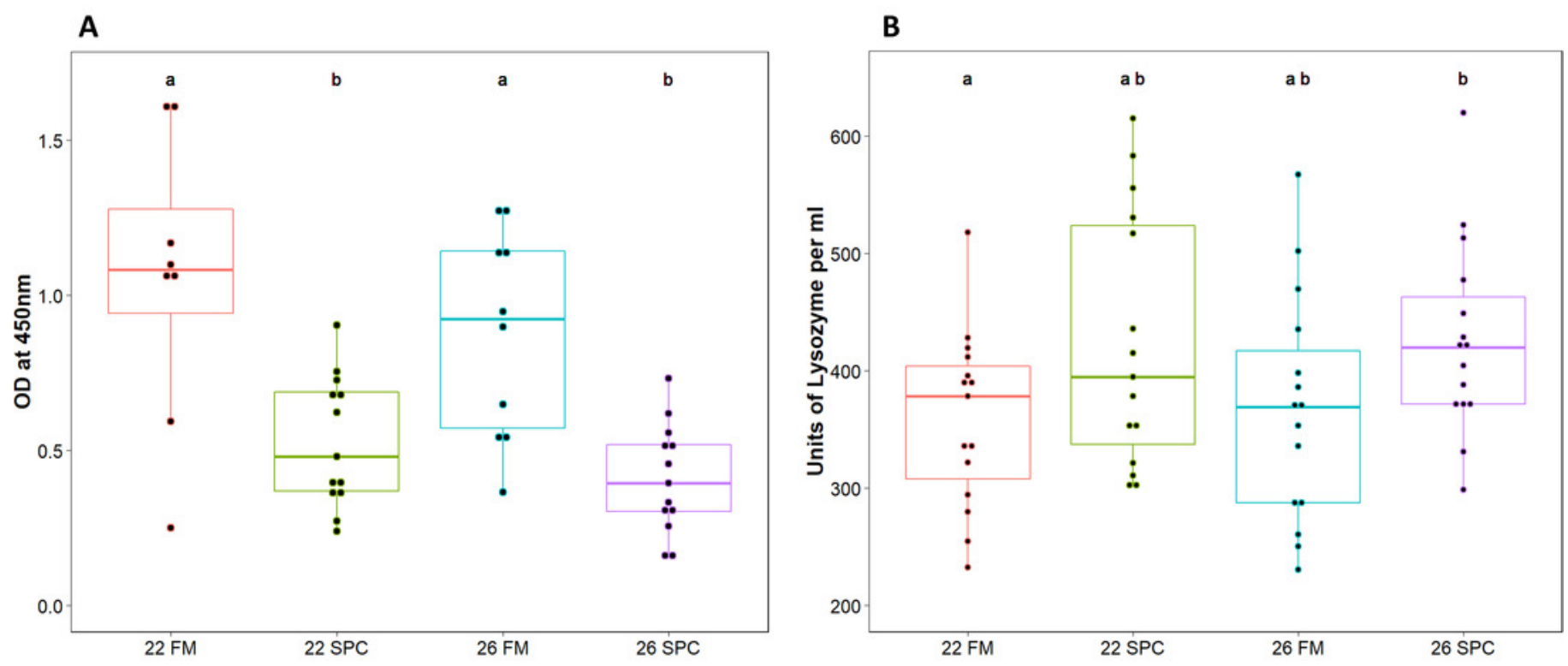


\section{Figure 2}

Alpha diversity (Shannon), richness (Chao1), and evenness (Simpson) of digesta, gut mucosa, skin, and water microbial communities.

Boxplots of (A) Chao1, (B) Shannon \& (C) Simpsons alpha diversity metrics for each sample, grouped by sampling site. Points represent individual samples and the box represents the median and interquartile range Different letters denote significant $(p<0.05)$ differences between treatments by ANOVA. 


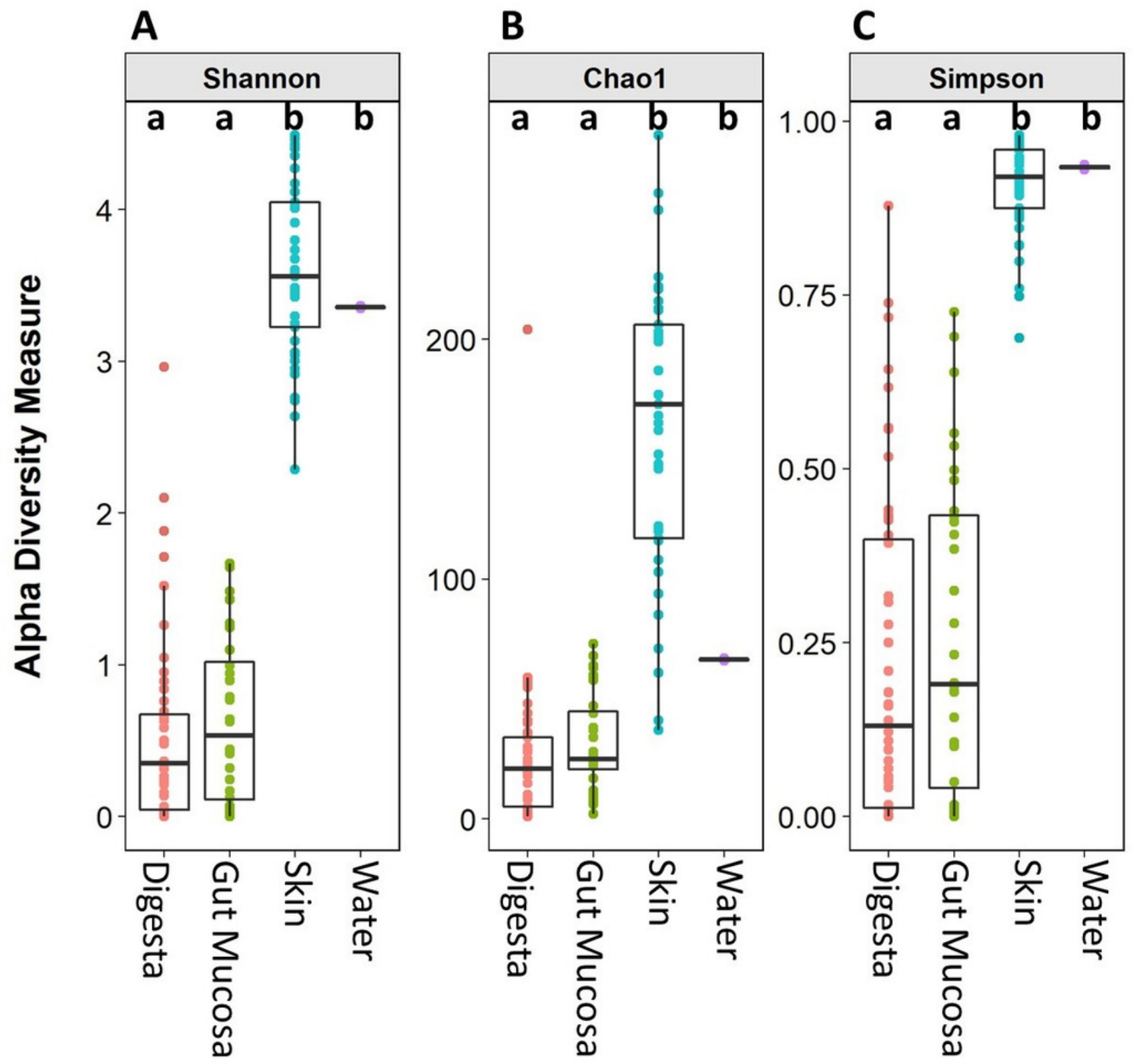




\section{Figure 3}

Distribution of OTUs identified in the gut mucosa, digesta, skin, and water samples.

Venn diagram detailing the number of OTUs that are unique to and shared between each of body sites and water.

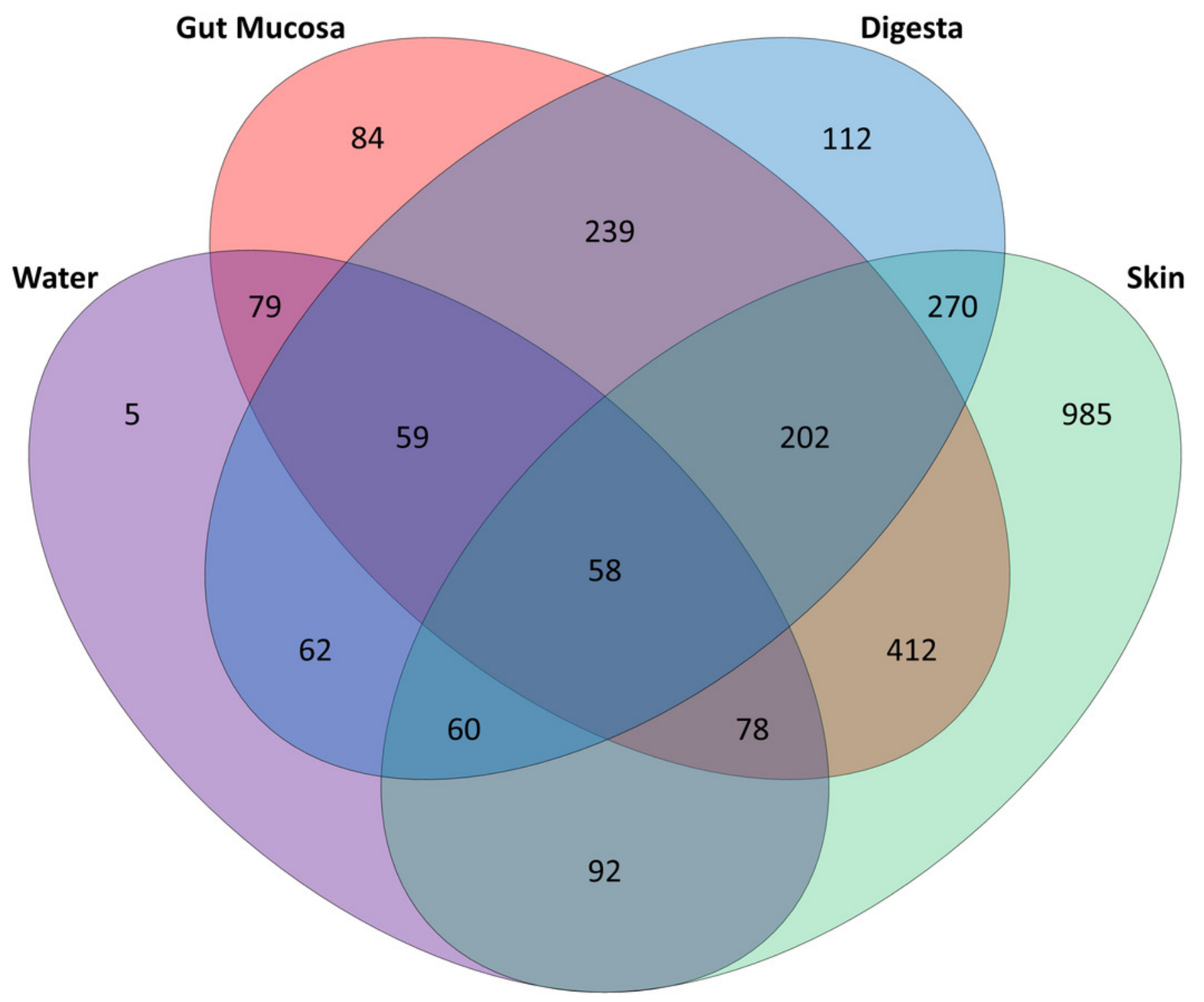




\section{Figure 4}

\section{Skin microbial composition (mean relative abundance of OTUs) for each treatment grouped by genus with assigned family detailed.}

Where no genus has been taxonomically assigned, the genus is left blank. Families that comprise less than $1 \%$ of the population are grouped together under remainder.

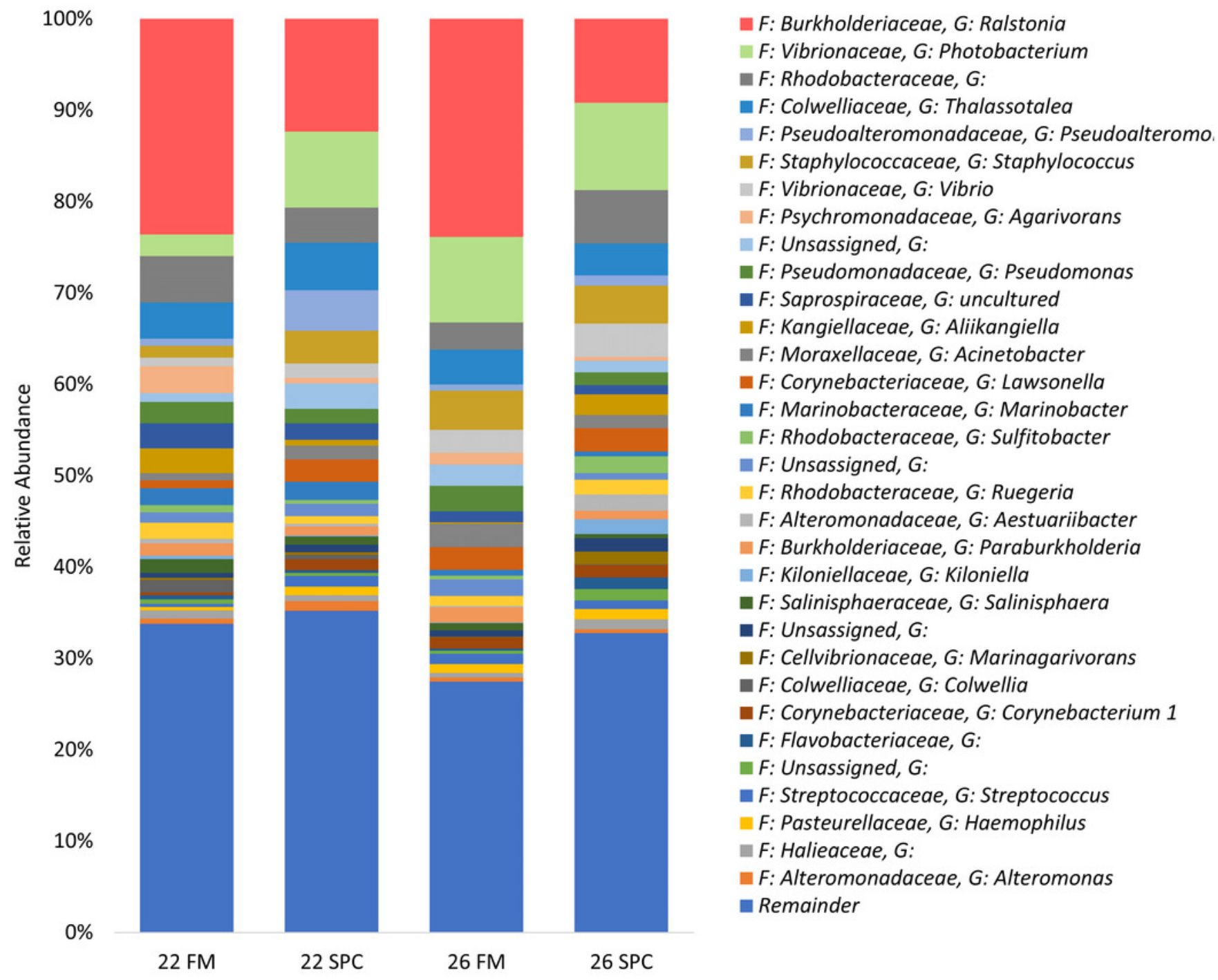




\section{Figure 5}

Impact of diet and temperature on the abundance of OTUs in the skin microbiome.

Differentially abundant OTUs were determined by LEfse analysis between control treatment and 22 SPC, 26 FM, and 26 SPC. The left histograms, (A), (C) and (D), show the significant ( $p$ $<0.05)$ LDA scores calculated for each OTU. The right heat maps, (B), (D) and (F), show the relative abundance ( $\log _{10}$ transformed) for each biological sample. Rows of the heat maps correspond to OTUs and columns to biological samples. Biological samples in the heat map are grouped by treatment and are labelled with their corresponding tank number. Blue and white denote the highest and lowest relative abundance respectively. OTUs were assigned at a genus level where possible, otherwise, the lowest inferred taxonomic level available was given. 
A

$\square 22 \mathrm{FM} \square 22 \mathrm{SPC}$

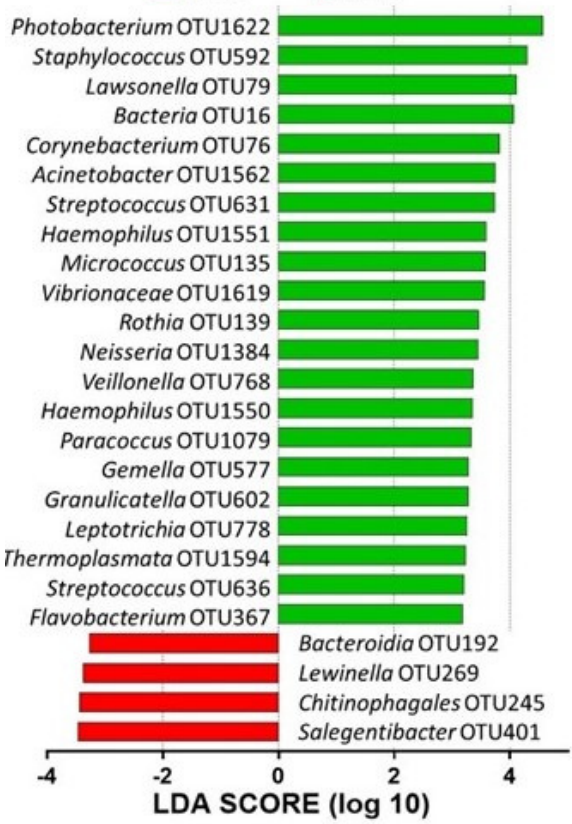

C

a $22 \mathrm{FM} \quad 26 \mathrm{FM}$

Photobacterium OTU1622 Staphylococcus OTU592

Lawsonella OTU79

Bacteria OTU16

Corynebacterium OTU76

Acinetobacter OTU1562

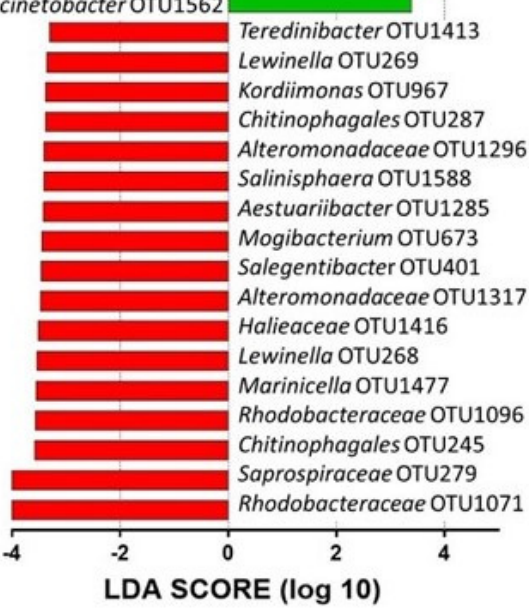

E

22 FM $26 \mathrm{SPC}$

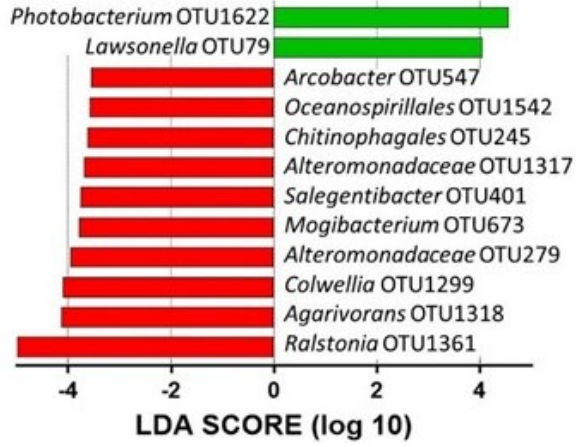

B

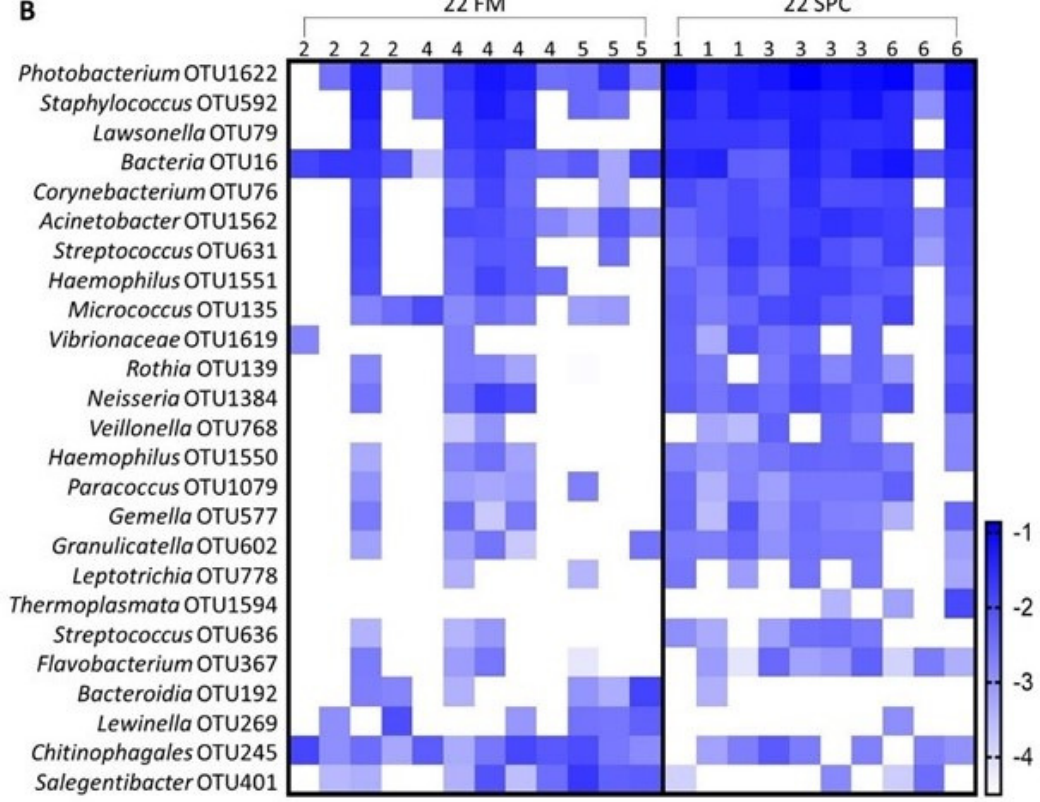

D

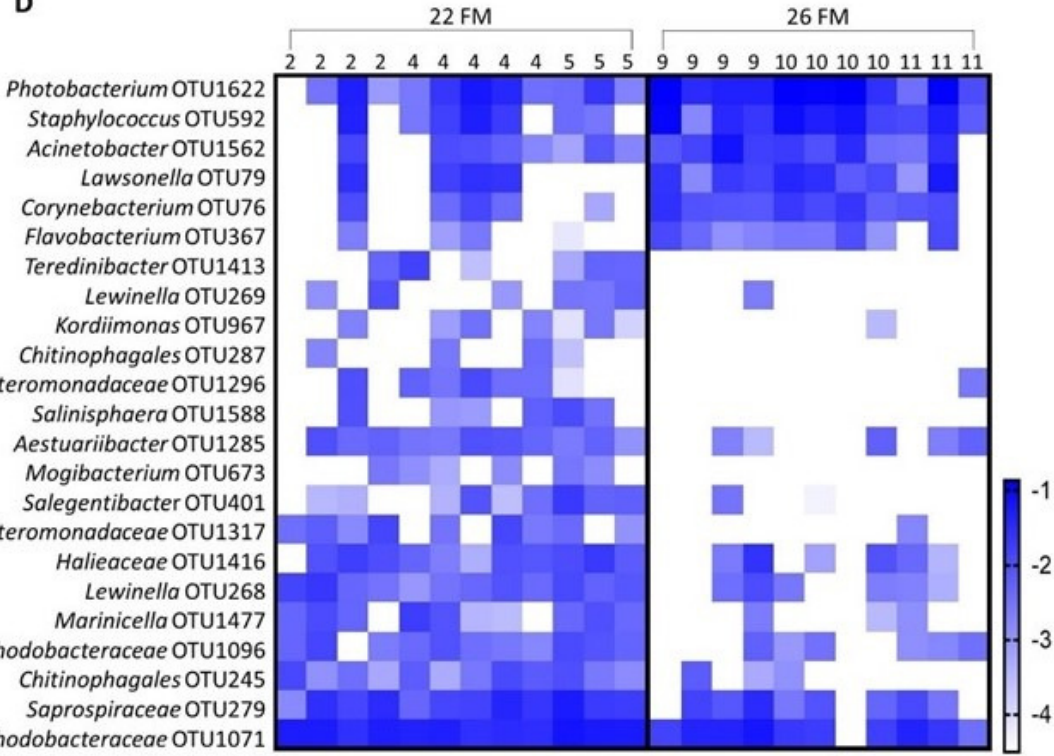

F

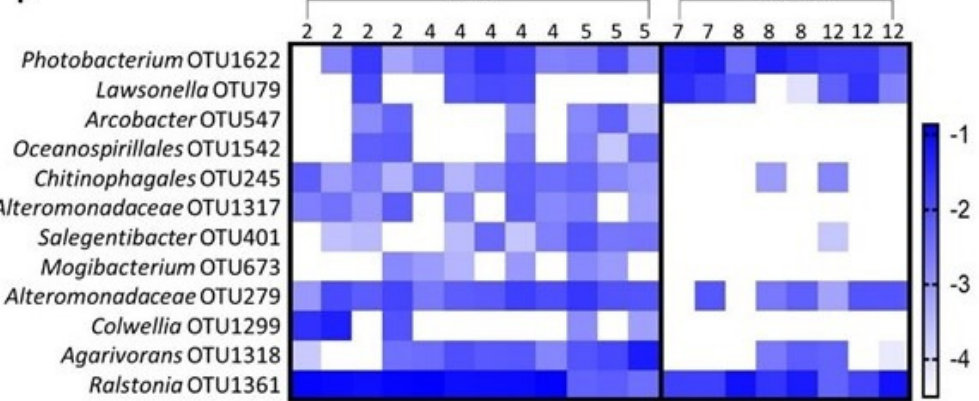




\section{Figure 6}

Digesta microbial composition (mean relative abundance of OTUs) for each treatment grouped by genus level with assigned family detailed.

Where no genus has been taxonomically assigned, the genus is left blank. Families that comprise less than $0.5 \%$ of the population in all treatments are grouped together under remainder.

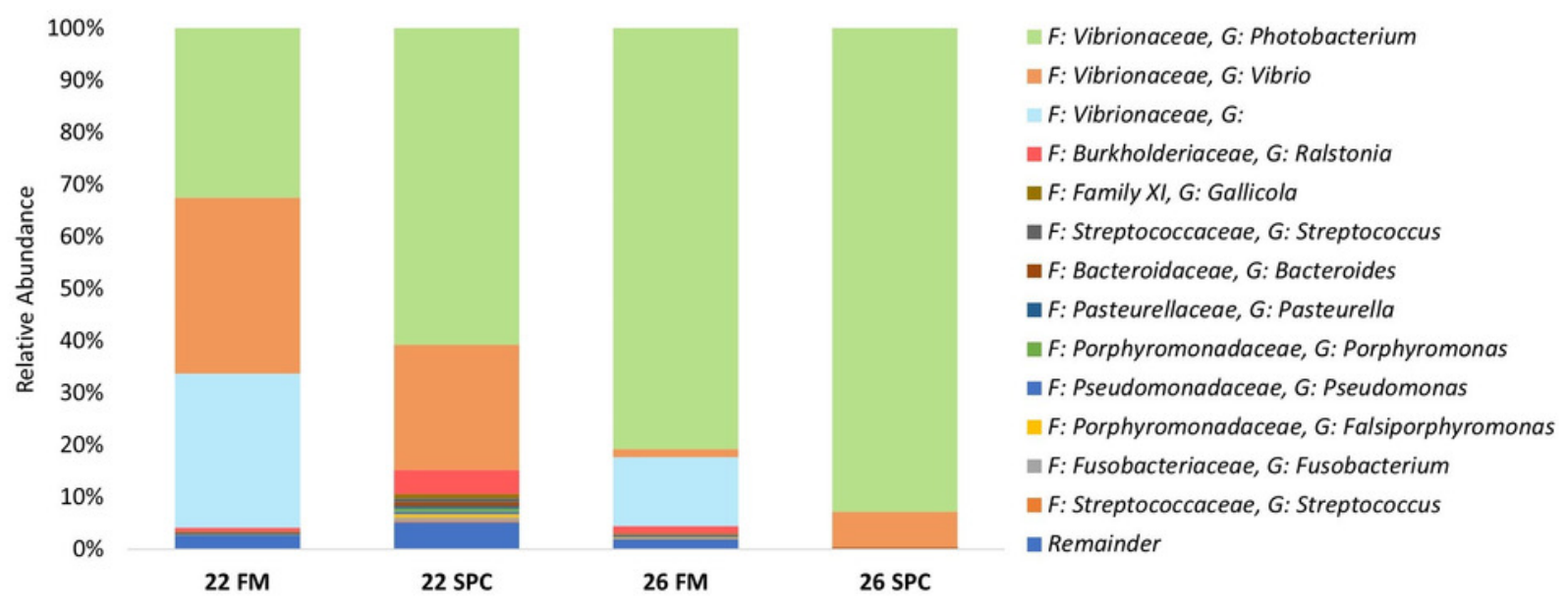




\section{Figure 7}

\section{Impact of diet and temperature on the abundance of OTUs in the digesta microbiome.}

Differentially abundant OTUs were determined by LEfse analysis between control treatment and 22 SPC, 26 FM, and 26 SPC. The left histograms, (A), (C) and (D), show the significant ( $p<0.05)$ LDA scores calculated for each OTU. The right heat maps, (B), (D) and (F), show the relative abundance ( $\log _{10}$ transformed) for each biological sample. Rows of the heat maps correspond to OTUs and columns to biological samples. Biological samples in the heat map are grouped by treatment and are labelled with their corresponding tank number. Blue and white denote the highest and lowest relative abundance respectively. OTUs were assigned at a genus level where possible, otherwise, the lowest inferred taxonomic level available was given.

A

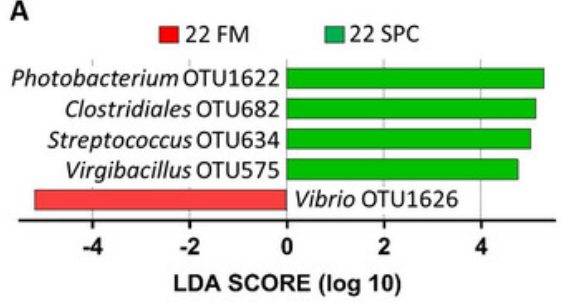

C

$\square 22 \mathrm{FM} \square 26 \mathrm{FM}$

Photobacterium OTU1622

Planctomycetes OTU831 Vibrionaceae OTU1619

$\begin{array}{lllll}-4 & -2 & 0 & 2 & 4\end{array}$

LDA SCORE $(\log 10)$

E

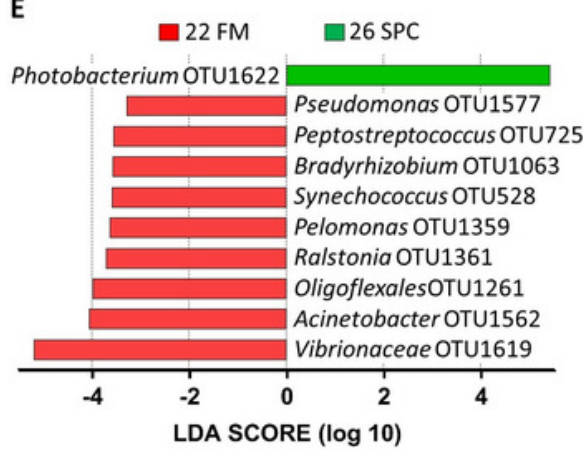

B

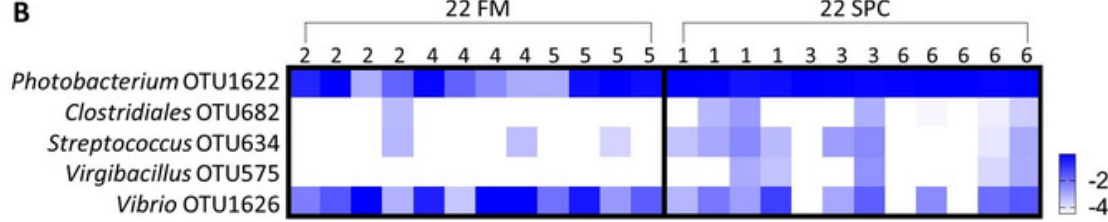

D

Photobacterium OTU1622

Planctomycetes OTU831

Vibrionaceae OTU1619

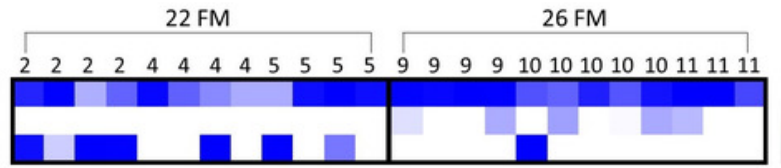

$-2$

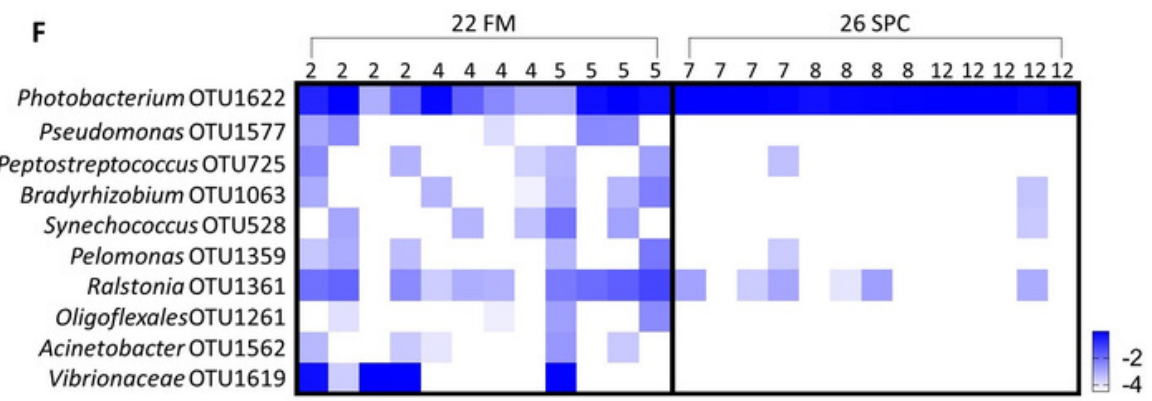




\section{Figure 8}

Graphical network representing the interactions in digesta samples between OTUs, diet, and digesta myeloperoxidase.

Only significant correlations $(p<0.05)$ are shown. Circular nodes correspond to OTUs and the size of these nodes represents the maximum relative abundance of that OTU across all digesta samples. Square nodes correspond to physiological measurements taken, and hexagonal nodes to diets. Red edges represent negative correlations, whilst blue edges represent positive correlations. The strength of the red or blue colour represents the strength of the correlation. Taxonomy assigned to OTUs is presented in Supplementary Table 4.

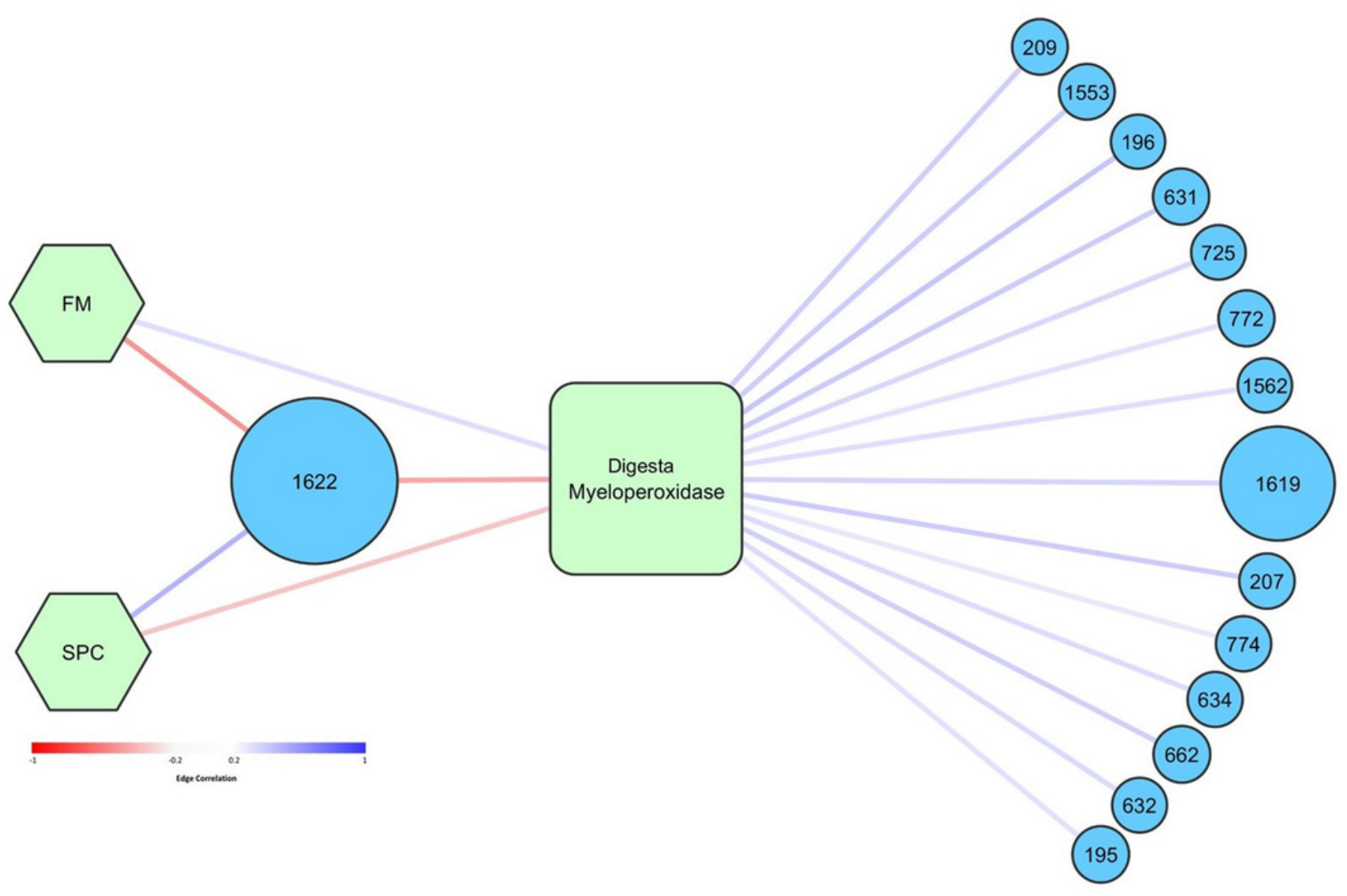




\section{Figure 9}

Mean relative abundance of OTUs present in gut mucosal samples grouped by genus.

(A) Mean relative abundance of OTUs in each treatment grouped by genera with family detailed. Genera comprising less than $0.2 \%$ grouped under remainder. (B) Mean relative abundance ( $\log 10$ transformed) of OTUs grouped by genera that comprise $>0.2 \%$ of each treatment. Rows of the heat map correspond to OTUs and columns to biological samples. Biological samples in the heat map are grouped by treatment and are labelled with their corresponding tank number. Blue and white denote the highest and lowest relative abundance respectively. Where no genus has been taxonomically assigned, the genus is left blank. 
A

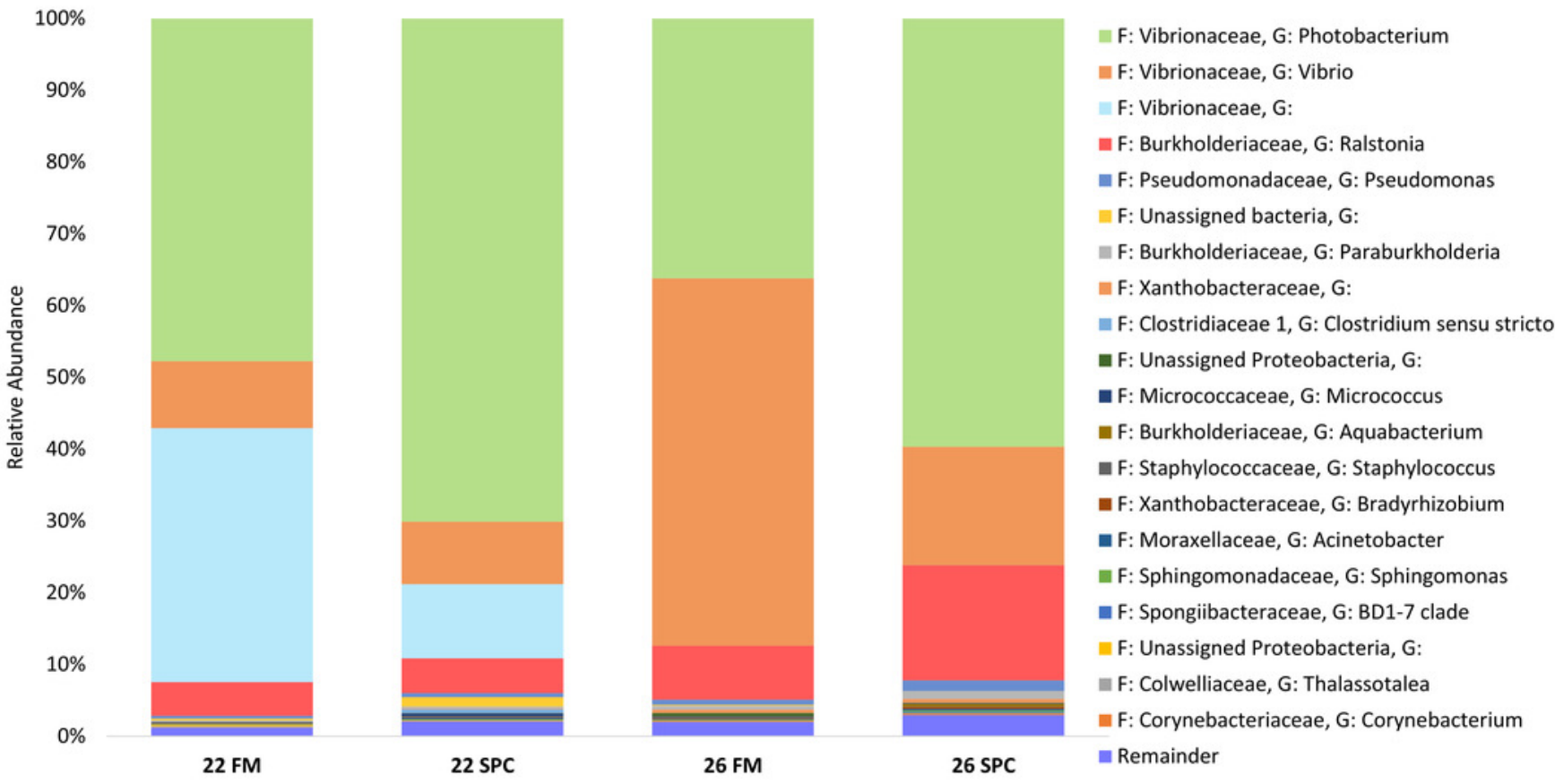

B

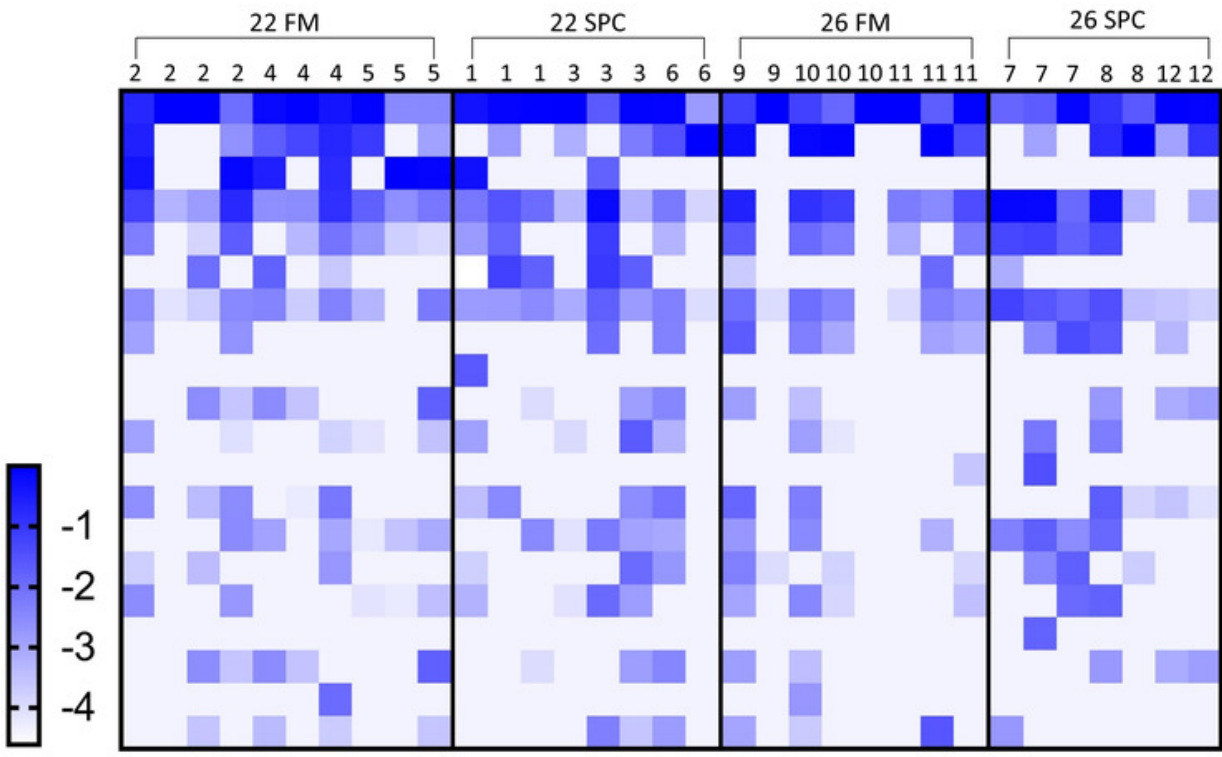

F: Vibrionaceae, G: Photobacterium F: Vibrionaceae, G: Vibrio

F: Vibrionaceae, G:

F: Burkholderiaceae, G: Ralstonia

F: Pseudomonadaceae, G: Pseudomonas

$\mathrm{F}$ : Unassigned bacteria, $\mathrm{G}$ :

F: Burkholderiaceae, G: Paraburkholderia

F: Xanthobacteraceae, G:

F: Clostridiaceae 1, G: Clostridium stricto

F: Unassigned Proteobacteria, G:

F: Micrococcaceae, G: Micrococcus

F: Burkholderiaceae, G: Aquabacterium

F: Staphylococcaceae, G: Staphylococcus

F: Xanthobacteraceae, G: Bradyrhizobium

F: Moraxellaceae, G: Acinetobacter

F: Sphingomonadaceae, G: Sphingomonas

F: Spongiibacteraceae, G: BD1-7 clade

F: Unassigned Proteobacteria, G:

F: Colwelliaceae, G: Thalassotalea

F: Corynebacteriaceae, G: Corynebacterium 


\section{Table $\mathbf{1}$ (on next page)}

Growth performance, feed conversion ratio, Fulton's body condition, and hepatosomatic index of yellowtail kingfish for each treatment.

${ }^{a}$ Means of triplicate tanks (5 fish per tank) \pm standard deviation. ${ }^{b}$ Specific growth rate (\% body weight per day $)=(\operatorname{In}($ final weight $)-\operatorname{In}($ initial weight $)) \times 100 /$ days ${ }^{c}$ Feed conversion ratio $=$ feed given $(\mathrm{g}) /$ weight gain $(\mathrm{g}) \cdot{ }^{\mathrm{d}}$ Fulton's body condition $=100 \times$ body weight $(\mathrm{g}) /$ /length $(\mathrm{cm}) \wedge$ 3. ${ }^{e}$ Hepatosomatic index $=100 \times$ liver weight $(\mathrm{g}) /$ body weight $(\mathrm{g})$. 


\begin{tabular}{|c|c|c|c|c|}
\hline Factors ${ }^{e}$ & $22 \mathrm{FM}$ & 22 SPC & $26 \mathrm{FM}$ & 26 SPC \\
\hline Weight gain $(\%)^{a}$ & $70.37 \% \pm 2.8 \%$ & $62.68 \% \pm 3.21 \%$ & $59.26 \% \pm 5.14 \%$ & $54.04 \% \pm 4.87 \%$ \\
\hline Weight gain $(g)^{a}$ & $201 \mathrm{~g} \pm 26 \mathrm{~g}$ & $181 \mathrm{~g} \pm 41 \mathrm{~g}$ & $173 g \pm 54 g$ & $155 g \pm 45 g$ \\
\hline $\operatorname{SGR}\left(\% \text { BW day }{ }^{-1}\right)^{\mathrm{b}}$ & $1.76 \pm 0.21$ & $1.64 \pm 0.20$ & $1.52 \pm 0.42$ & $1.41 \pm 0.41$ \\
\hline Fork length growth $(\%)^{a}$ & $17.38 \% \pm 0.76 \%$ & $16.72 \% \pm 1.57 \%$ & $13.35 \% \pm 0.97 \%$ & $13.07 \% \pm 1.54 \%$ \\
\hline Feed conversion ratio ${ }^{c}$ & $0.93 \pm 0.02$ & $0.99 \pm 0.02$ & $1.09 \pm 0.06$ & $1.19 \pm 0.04$ \\
\hline Fulton's body condition ${ }^{d}$ & $1.43 \pm 0.03$ & $1.42 \pm 0.01$ & $1.45 \pm 0.01$ & $1.49 \pm 0.02$ \\
\hline Hepatosomatic index ${ }^{e}$ & $1.07 \pm 0.04$ & $1.03 \pm 0.03$ & $1.18 \pm 0.04$ & $1.16 \pm 0.03$ \\
\hline
\end{tabular}

1 


\section{Table 2 (on next page)}

Sample numbers, sequencing reads, and OTU statistics post quality control filtering for fish body sites and filtered estuarine water ${ }^{a}$

${ }^{a}$ Number of samples, total reads, mean reads ( $\left.\pm S D\right)$, total OTUs, and unique OTUs for the gut mucosa, digesta, skin, and water samples. 


\begin{tabular}{ccccccc}
\hline $\begin{array}{c}\text { Sampling } \\
\text { Site }\end{array}$ & $\begin{array}{c}\text { Sampling } \\
\text { Time Point }\end{array}$ & $\begin{array}{c}\text { Baseline } \\
\text { Samples }\end{array}$ & Total Reads & $\begin{array}{c}\text { Average reads } \\
\text { per sample }\end{array}$ & Total OTUs & $\begin{array}{c}\text { Unique } \\
\text { OTUs }\end{array}$ \\
\hline \multirow{3}{*}{ Gut Mucosa } & Baseline & 15 & 886,485 & $59,099 \pm 24,628$ & 388 & \\
& Final & 33 & $2,097,015$ & $63,545 \pm 32,000$ & 318 & \\
\cline { 2 - 7 } & Total & 48 & $2,983,500$ & $62,156 \pm 29,702$ & 531 & 84 \\
\cline { 2 - 7 } Digesta & Baseline & 16 & 485,621 & $44,147 \pm 4,482$ & 162 & \\
& Final & 43 & $2,320,220$ & $48,337 \pm 11,925$ & 355 & \\
\cline { 2 - 7 } & Total & 59 & $2,805,841$ & $47,556 \pm 11,019$ & 418 & 112 \\
\cline { 2 - 7 } Skin & Baseline & 15 & 706,277 & $47,085 \pm 15,650$ & 973 & \\
& Final & 41 & $2,025,615$ & $49,405 \pm 20,197$ & 1,232 & \\
\cline { 2 - 7 } & Total & 56 & $2,731,892$ & $48,784 \pm 18,976$ & 1,476 & 985 \\
\cline { 2 - 7 } $\begin{array}{c}\text { Rearing } \\
\text { Water }\end{array}$ & Final & 2 & & & & \\
\hline TOTAL & & $\mathbf{6 6 6 , 7 4 1}$ & $33,371 \pm 4,060$ & 97 & 5 \\
\hline
\end{tabular}

1 


\section{Table 3 (on next page)}

Pairwise PERMANOVA comparing the microbial communities of gut mucosa, digesta, and skin samples by treatment ${ }^{a}$

${ }^{a}$ Pairwise PERMANOVA with 999 permutations was performed on a Bray-Curtis dissimilarity matrix. Comparisons for which $p<0.05$ are presented in bold. 


\begin{tabular}{cc|cc|cc|cc|cc}
\hline & & \multicolumn{2}{|c|}{22 FM } & \multicolumn{2}{c|}{22 SPC } & \multicolumn{2}{c}{ 26 FM } & \multicolumn{2}{c}{ 26 SPC } \\
Body site & Body Site & $\mathbf{R}^{\mathbf{2}}$ & $\mathbf{p}$ & $\mathbf{R}^{\mathbf{2}}$ & $\mathbf{p}$ & $\mathbf{R}^{\mathbf{2}}$ & $\mathbf{p}$ & $\mathbf{R}^{\mathbf{2}}$ & $\mathbf{p}$ \\
\hline Gut Mucosa & Digesta & 0.083 & 0.125 & 0.124 & $\mathbf{0 . 0 2 5}$ & 0.053 & 0.428 & 0.217 & $\mathbf{0 . 0 0 2}$ \\
Gut Mucosa & Skin & 0.285 & $\mathbf{0 . 0 0 1}$ & 0.231 & $\mathbf{0 . 0 0 1}$ & 0.181 & $\mathbf{0 . 0 0 2}$ & 0.138 & $\mathbf{0 . 0 0 2}$ \\
Digesta & Skin & 0.255 & $\mathbf{0 . 0 0 1}$ & 0.408 & $\mathbf{0 . 0 0 1}$ & 0.252 & $\mathbf{0 . 0 0 2}$ & 0.435 & $\mathbf{0 . 0 0 1}$ \\
\hline
\end{tabular}

1 


\section{Table 4 (on next page)}

Pairwise PERMANOVA comparing the microbial communities of experimental treatments to the control in the skin ${ }^{a}$

${ }^{a}$ Pairwise PERMANOVA with 999 permutations was performed on a Bray-Curtis dissimilarity matrix. Comparisons for which $p<0.05$ are presented in bold. 


\begin{tabular}{lllll}
\hline Control & Treatment & Differ by & $\mathbf{R}^{\mathbf{2}}$ & $\mathbf{p}$ \\
\hline $22 \mathrm{FM}$ & $22 \mathrm{SPC}$ & Diet & 0.107 & $\mathbf{0 . 0 0 5}$ \\
$22 \mathrm{FM}$ & $26 \mathrm{FM}$ & Temp & 0.083 & $\mathbf{0 . 0 0 8}$ \\
$22 \mathrm{FM}$ & $26 \mathrm{SPC}$ & Diet + Temp & 0.117 & $\mathbf{0 . 0 0 3}$ \\
\hline
\end{tabular}

1 


\section{Table 5 (on next page)}

Pairwise PERMANOVA comparing the microbial communities of experimental treatments to the control in the digesta ${ }^{a}$

${ }^{a}$ Pairwise PERMANOVA with 999 permutations was performed on a Bray-Curtis dissimilarity matrix. Comparisons for which $p<0.05$ are presented in bold. 
1

\begin{tabular}{lllll}
\hline Control & Treatment & Differ by & $\mathbf{R}^{\mathbf{2}}$ & $\mathbf{p}$ \\
\hline $22 \mathrm{FM}$ & $22 \mathrm{SPC}$ & Diet & 0.173 & $\mathbf{0 . 0 0 5}$ \\
$22 \mathrm{FM}$ & $26 \mathrm{FM}$ & Temp & 0.118 & $\mathbf{0 . 0 2 9}$ \\
$22 \mathrm{FM}$ & $26 \mathrm{SPC}$ & Diet + Temp & 0.267 & $\mathbf{0 . 0 0 1}$ \\
\hline
\end{tabular}

